Pacific

Journal of

Mathematics

RIBAUCOUR TRANSFORMATIONS FOR CONSTANT MEAN CURVATURE AND LINEAR WEINGARTEN SURFACES

A.V. Corro, W. Ferreira, and K. Tenenblat 


\title{
RIBAUCOUR TRANSFORMATIONS FOR CONSTANT MEAN CURVATURE AND LINEAR WEINGARTEN SURFACES
}

\author{
A.V. Corro, W. Ferreira, and K. Tenenblat
}

We provide a method to obtain linear Weingarten surfaces from a given such surface, by imposing a one parameter algebraic condition on a Ribaucour transformation. Our main result extends classical results for surfaces of constant Gaussian or mean curvature. By applying the theory to the cylinder, we obtain a two-parameter family of complete linear Weingarten surfaces (hyperbolic, elliptic and tubular), asymptotically close to the cylinder, which have constant mean curvature when one of the parameters vanishes. The family contains $n$-bubble Weingarten surfaces which are 1-periodic, have genus zero and two ends of geometric index $m$, where $n / m$ is an irreducible rational number. Their total curvature vanishes, while the total absolute curvature is $8 \pi n$. We also apply the method to obtain families of complete constant mean curvature surfaces, associated to the Delaunay surfaces, which are 1-periodic for special values of the parameter.

\section{Introduction.}

In the last two decades a great activity in research has been devoted to surfaces of constant mean curvature (cmc) surfaces. After the first example of a non-totally umbilical compact cmc surface immersed in $R^{3}$ found by Wente $[\mathbf{W} \mathbf{1}]$, a series of papers by Meeks $[\mathbf{M}]$, Korevaar, Kusner and Solomon [KKS], Pinkal and Sterling [PS], Kapouleas [K], Karcher [Ka], Abresch $[\mathbf{A}]$, Walter $[\mathbf{W a}]$ gave important contributions to the theory and to the construction of examples of complete immersed cmc surfaces.

Some of the results proved for cmc surfaces were also extended by Rosenberg and Sa Earp $[\mathbf{R S}]$, and by Brito and Sa Earp [BS] to the so called special Weingarten immersed surfaces, and in particular to surfaces whose mean curvature $H$ and Gaussian curvature $K$ satisfy a linear relation $a H+K=b$ where $a \geq 0, b>0$.

In this paper, we consider a method of constructing linear Weingarten surfaces based on Ribaucour transformations. A linear Weingarten surface of $R^{3}$ is a surface whose Gaussian curvature $K$ and mean curvature $H$ satisfy a linear relation $\alpha+\beta H+\gamma K=0$, where $\alpha, \beta, \gamma \in R$. Such a surface is said 
to be hyperbolic (resp. elliptic) when $\Delta:=\beta^{2}-4 \alpha \gamma<0$ (resp. $\Delta>0$ ). The relation $\Delta=0$ characterizes the tubular surfaces. In particular, surfaces of constant negative Gaussian curvature are hyperbolic, while surfaces of constant mean curvature (including minimal) and constant positive curvature are elliptic. Ribaucour transformations for constant Gaussian curvature and constant mean curvature surfaces, were considered at the beginning of last century (see Bianchi $[\mathbf{B i}]$ ) and they were recently applied for the first time to obtain minimal surfaces [CFT2]. We should mention that Bäcklund transformations for hyperbolic linear Weingarten surfaces were considered by $[\mathbf{B i}]$ and generalized to higher dimensions by $[\mathbf{B u}]$.

Our main result in this paper extends Ribaucour transformations to linear Weingarten surfaces and also provides a unified version for the classical results. As an application of the theory, we obtain an interesting, two-parameter family of complete linear Weingarten surfaces associated to the cylinder. This family shows that the Ribaucour transformation under consideration is not necessarily a Darboux transformation and it provides an unexpected result. Namely, the existence of complete hyperbolic linear Weingarten surfaces immersed in $R^{3}$. Hilbert's theorem shows that there are no complete surfaces of constant negative curvature immersed in $R^{3}$. Although such surfaces and hyperbolic linear Weingarten surfaces correspond to solutions of the sine-Gordon equation, the family of examples associated to the cylinder shows that there exist infinitely many complete hyperbolic linear Weingarten surfaces immersed in $R^{3}$. The results of this paper were announced in $[\mathbf{T}]$.

We point out that although linear Weingarten surfaces are locally parallel to minimal surfaces or surfaces of constant Gaussian curvature, with same $\Delta$, the parallelism procedure cannot be applied to extend the method of Ribaucour transformation to linear Weingarten surfaces, since it is not a global construction and in general it produces singularities. This is reinforced by the existence of complete hyperbolic linear Weingarten surfaces, which obviously cannot be parallel to any complete surface of constant negative Gaussian curvature.

The paper is organized as follows: In Section 1, we consider Ribaucour transformations for surfaces and we provide an algebraic condition for such a transformation to relate two linear Weingarten surfaces. We show that the linear system of differential equations corresponding to the Ribaucour transformation with the additional algebraic condition is integrable on linear Weingarten surfaces. As a consequence of the theory we get the corresponding results for $H$-cmc surfaces. In this case, for a given such surface, the parameter must satisfy $c(c-2 H)>0$.

In Section 2, by considering the cylinder as a linear Weingarten surface satisfying $-1 / 2+H+\gamma K=0$, we obtain a two-parameter family of immersions $\tilde{X}_{c \gamma}$, of $R^{2}$ into euclidean space $R^{3}$, which are linear Weingarten 
surfaces. Not all of them are complete. We characterize the complete ones in terms of the pair of real numbers $(c, \gamma)$. This family of complete surfaces contains hyperbolic, elliptic and tubular linear Weingarten surfaces which are all asymptotically close to the cylinder. One family of lines of curvature is planar while the other one is spherical (which may degenarate into planar). We describe the symmetries of these surfaces, which are quite distinct whenever $c<0$ and $c>0$. For generic values of the parameters, the immersions are not periodic. However, for special values of $(c, \gamma)$, namely for each $c<0$ and $\sqrt{1-c(2 \gamma+1)}=n / m$ an irreducible rational number, we get an $n$-bubble surface $\widetilde{X}_{c \gamma}$. This is an immersed cylinder into $R^{3}$, with two ends of geometric index $m$ and $n$ isolated points of maximum and of minimum for the Gaussian curvature. We show that its total curvature vanishes while its total absolute curvature is $8 \pi n$. Moreover, we prove that the ends are embedded if and only if $m=1$ and in this case, they are cylindrical ends. If $c>0$ or $c<0$ and $\sqrt{1-c(2 \gamma+1)}$ is not a rational number, then $\widetilde{X}_{c \gamma}$ is a complete immersion of $R^{2}$ into $R^{3}$, not periodic in any variable and it has an infinite number of isolated critical points for the Gaussian curvature.

In Section 3, by restricting the constant $\gamma=0$ in the previous section, we get a one parameter family of complete $1 / 2$-cmc immersions $\widetilde{X}_{c}$ from $R^{2}$ into $R^{3}$. These surfaces are not periodic for generic values of $c$. However, special values of $c$, produce 1 -periodic cmc $n$-bubble surfaces. These immersed cmccylinders were first described by Sievert $[\mathbf{S}]$ for $n=2$ (see also $[\mathbf{P S}]$ ), and their existence was proved later in $[\mathbf{G}-\mathbf{B}]$ and $[\mathbf{S W}]$. We also show that these surfaces are of finite type one (as defined in $[\mathbf{P S}]$ ).

In Section 4, we obtain families of cmc surfaces associated to the Delaunay surface by Ribaucour transformations. By restricting the range of the parameter $c$ conveniently we obtain families of complete $H$-cmc surfaces. We describe their symmetries and as in the case of the surfaces associated to the cylinder, for special values of $c$ we get a family of 1-periodic surfaces.

\section{Ribaucour transformation for linear Weingarten surfaces.}

In this section, we first recall the theory of Ribaucour transformation for surfaces. For the proofs and more details see [Bi] and [CFT1]. We then prove that, imposing a one-parameter algebraic condition on a Ribaucour transformation, we have a correspondence between linear Weingarten surfaces. We show that starting with such a surface the system of equations is integrable and provides a family of new Weingarten surfaces. As a consequence of the theory we get the corresponding results for $\mathrm{cmc}$ surfaces.

Let $M$ be an orientable surface of $R^{3}$ without umbilic points. We denote by $N$ its Gauss map. We say that $\widetilde{M}$ is associated by a Ribaucour transformation to $M$, if and only if, there exists a differentiable function $h$ defined on $M$ and a diffeomorphism $\psi: M \rightarrow \widetilde{M}$ such that: $p+h(p) N(p)=$ 
$\psi(p)+h(p) \widetilde{N}(\psi(p))$, for all $p \in M$, where $\widetilde{N}$ is the normal map of $\widetilde{M}$; the subset $p+h(p) N(p), p \in M$, is an 2-dimensional submanifold and $\psi$ preserves lines of curvature.

We say that $\widetilde{M}$ is locally associated by a Ribaucour transformation to $M$ if for all $\widetilde{p} \in \widetilde{M}$ there exists a neighborhood of $\widetilde{p}$ in $\widetilde{M}$ which is associated by a Ribaucour transformation to an open subset of $M$. Similarly, one may consider the corresponding definitions for parametrized surfaces.

The following results give a characterization of Ribaucour transformations.

Theorem 1.1. Let $M$ be an orientable surface of $R^{3}$, without umbilic points and $N$ its Gauss map. Let $e_{i}, 1 \leq i \leq 2$ be orthonormal principal directions, $\lambda^{i}$ the corresponding principal curvatures, i.e., $d N\left(e_{i}\right)=\lambda_{i} e_{i}$. A surface $\widetilde{M}$ is locally associated to $M$ by a Ribaucour transformation, if and only if, there exist parametrizations $\widetilde{X}: U \subset R^{2} \rightarrow \widetilde{M}$ and $X: U \subset R^{2} \rightarrow M$ and a differentiable function $h: U \rightarrow R$ such that $1+h \lambda^{i} \neq 0$,

$$
\widetilde{X}=X+h(N-\tilde{N}),
$$

where $\tilde{N}$ is a unit vector field normal to $\tilde{X}(U)$ given by

$$
\begin{gathered}
\tilde{N}=\frac{1}{\Delta+1}\left(\sum_{i=1}^{n} 2 Z^{i} e_{i}+(\Delta-1) N\right), \\
Z^{i}=\frac{d h\left(e_{i}\right)}{1+h \lambda^{i}} \quad \Delta=\sum_{i=1}^{n}\left(Z^{i}\right)^{2}
\end{gathered}
$$

and $h$ is a generic solution of the differential equation

$$
d Z^{j}\left(e_{i}\right)+Z^{i} \omega_{i j}\left(e_{i}\right)-Z^{i} Z^{j} \lambda^{i}=0, \quad 1 \leq i \neq j \leq 2,
$$

where $\omega_{i j}$ are the connection forms of the frame $e_{i}$.

Proof. Let $\tilde{N}$ be a unit vector field given by

$$
\widetilde{N}=\sum_{i=1}^{2} b^{i} e_{i}+b^{3} N, \quad \text { where } \quad \sum_{i=1}^{2}\left(b^{i}\right)^{2}+\left(b^{3}\right)^{2}=1 .
$$

We introduce the following notation:

$$
d \tilde{N}\left(e_{i}\right)=\sum_{k=1}^{2} L_{i}^{k} e_{k}+L_{i}^{3} N
$$

where for $1 \leq i, k \leq 2$

$$
L_{i}^{k}=d b^{k}\left(e_{i}\right)+\sum_{j} b^{j} \omega_{j k}\left(e_{i}\right)+b^{3} \lambda^{i} \delta_{i k}, \quad L_{i}^{3}=d b^{3}\left(e_{i}\right)-b^{i} \lambda^{i} .
$$


We will later show that the following relations hold:

$$
b^{i}=Z^{i}\left(1-b^{3}\right) \text {. }
$$

In this case, from (5) we get

$$
b^{3}=\frac{\Delta-1}{\Delta+1} .
$$

We will now prove the theorem. Assume that $\widetilde{M}$ is locally associated to $M$ by a Ribaucour transformation. Then by definition there exist local parametrizations $X$ of $M, \widetilde{X}$ of $\widetilde{M}$ and a function $h$ defined on $U \subset R^{2}$ such that $\widetilde{X}+h \widetilde{N}=X+h N$, where $\widetilde{N}$ is a unit vector field normal to $\widetilde{M}$, which may be considered as in (5). Since

$$
d \widetilde{X}=d X+d h(N-\widetilde{N})+h(d N-d \widetilde{N})
$$

it follows from the relations $d X=\sum_{j} \omega_{j} e_{j}$ and $d N\left(e_{i}\right)=\lambda^{i} e_{i}$ that

$$
d \widetilde{X}\left(e_{i}\right)=\left(1+h \lambda^{i}\right) e_{i}+d h\left(e_{i}\right)(N-\widetilde{N})-h d \widetilde{N}\left(e_{i}\right) .
$$

Hence, $\left\langle d \widetilde{X}\left(e_{i}\right), \tilde{N}\right\rangle=0$ implies

$$
\left(1+h \lambda^{i}\right) b^{i}+d h\left(e_{i}\right)\left(b^{3}-1\right)=0, \quad i=1,2 .
$$

Since $X+h N$ is two dimensional, it follows that $1+h \lambda^{i} \neq 0$ for all $i$. Therefore we conclude from (12) that the relations (8) hold and hence $b^{3}$ is given by $(9) . d \widetilde{X}\left(e_{i}\right)$ are orthogonal principal directions, i.e.,

$$
\left\langle d \widetilde{X}\left(e_{i}\right), d \widetilde{X}\left(e_{j}\right)\right\rangle=\left\langle d \widetilde{N}\left(e_{j}\right), d \widetilde{X}\left(e_{i}\right)\right\rangle=\left\langle d \widetilde{N}\left(e_{j}\right), d \widetilde{N}\left(e_{i}\right)\right\rangle=0 \quad \text { for } i \neq j .
$$

Hence, using (6) and Equation (11), we get

$$
\left\langle d \widetilde{N}\left(e_{i}\right), d \widetilde{X}\left(e_{j}\right)\right\rangle=L_{i}^{j}\left(1+h \lambda^{j}\right)+L_{i}^{3} d h\left(e_{j}\right)=0, \quad \text { for } i \neq j .
$$

It follows from (7) and the last equality, that $h$ satisfies Equation (4).

Conversely, assume $h$ is a solution of (4) such that $1+h \lambda^{i} \neq 0, \forall i$, then we define $Z^{i}$ and $\Delta$ by (3), $b^{i}$ and $b^{3}$ by (8) and (9). It follows from (7) and (4) that

$$
L_{i}^{k}+Z^{k} L_{i}^{3}=0, \quad i \neq k,
$$

and

$$
Z^{i} L_{i}^{i}+\left(\left(Z^{i}\right)^{2}-\frac{\Delta+1}{2}\right) L_{i}^{3}=0
$$

We consider $\tilde{N}$ and $\tilde{X}$ as in (5) and (1) respectively. We need to show that $\widetilde{X}$ is associated to $X$ by a Ribaucour transformation. We first observe that $\widetilde{N}$ is a unit vector field. In fact, $\sum_{i}\left(b^{i}\right)^{2}+\left(b^{3}\right)^{2}=\left(1-b^{3}\right)^{2} \Delta+\left(b^{3}\right)^{2}=1$, since $b^{3}$ is given by (9). We next verify that $\widetilde{N}$ is normal to $\widetilde{X}$. From the definition of 
$\widetilde{X}$, we have that $d \widetilde{X}\left(e_{i}\right)$ is given by (11). Hence, using the fact that $|\tilde{N}|=1$, we conclude that $\left\langle d \widetilde{X}\left(e_{i}\right), \widetilde{N}\right\rangle=\left(-\left(1+h \lambda^{i}\right) Z^{i}+d h\left(e_{i}\right)\right)\left(b^{3}-1\right)=0$.

Using (6), Equation (14) and the definition of $\Delta$, one proves that $\left\langle d \widetilde{N}\left(e_{1}\right)\right.$, $\left.d \widetilde{N}\left(e_{2}\right)\right\rangle=0$. Therefore, it follows from Equations (10) and (13), that for $i \neq j$

$$
\left\langle d \widetilde{N}\left(e_{i}\right), d \widetilde{X}\left(e_{j}\right)\right\rangle=L_{i}^{j}\left(1+h \lambda^{j}\right)+L_{i}^{3} d h\left(e_{j}\right)=0 .
$$

Finally, we prove that $d \widetilde{X}\left(e_{1}\right)$ and $d \widetilde{X}\left(e_{2}\right)$ are orthogonal. In fact,

$$
\left\langle d \tilde{X}\left(e_{1}\right), d \widetilde{X}\left(e_{2}\right)\right\rangle=d h\left(e_{2}\right)\left[-\left(1+h \lambda^{1}\right) b^{1}+d h\left(e_{1}\right)\left(1-b^{3}\right)\right]=0,
$$

where the last equality follows from the definition of $b^{i}$ and Equation (13). Moreover, for generic $h, \widetilde{X}$ is an immersed surface. Introducing the eigenvalues $\widetilde{\lambda}^{i}, d \widetilde{N}\left(e_{i}\right)=\widetilde{\lambda}^{i} d \widetilde{X}\left(e_{i}\right)$, it follows from (11) that

$$
\left(1+h \widetilde{\lambda}^{i}\right) d \widetilde{X}\left(e_{i}\right)=\left(1+h \lambda^{i}\right) e_{i}+d h\left(e_{i}\right)(N-\widetilde{N}) .
$$

Hence we conclude from (5) and (8) that

$$
\left|1+h \widetilde{\lambda}^{i}\right|\left|d \widetilde{X}\left(e_{i}\right)\right|=\left|1+h \lambda^{i}\right| .
$$

One can linearize Equation (4) as we will show in Proposition 1.2. We first observe that from the proof given above and from (6), we have $\left\langle d \widetilde{N}\left(e_{i}\right), N\right\rangle=$ $L_{i}^{3}=\widetilde{\lambda}^{i}\left\langle d \widetilde{X}\left(e_{i}\right), N\right\rangle$. Therefore, whenever $d h\left(e_{i}\right) \neq 0$, using (11), (5) and (6) we obtain the principal curvatures given by

$$
\tilde{\lambda}^{i}=\frac{L_{i}^{3}}{d h\left(e_{i}\right)\left(1-b^{3}\right)-h L_{i}^{3}} .
$$

Proposition 1.2. If $h$ is a solution of (4) which does not vanish on a simply connected domain, then $h=\Omega / W$ where $\Omega$ and a non-vanishing function $W$ satisfy

$$
\begin{aligned}
d \Omega_{i}\left(e_{j}\right) & =\Omega_{j} \omega_{i j}\left(e_{j}\right), \quad \text { for } i \neq j, \\
d \Omega & =\sum_{i=1}^{2} \Omega_{i} \omega_{i}, \\
d W & =-\sum_{i=1}^{2} \Omega_{i} \lambda^{i} \omega_{i} .
\end{aligned}
$$

Conversely, suppose (16)-(18) are satisfied, such that $W\left(W+\Omega \lambda^{i}\right) \neq 0$, then $h=\Omega / W$ is a solution of (4).

Proof. Assume $h$ is a nonvanishing solution of (4), then $\psi=\sum_{i} Z^{i} \omega_{i} / h$, is a closed form. Hence, on a simply connected domain there exists a differentiable function $\Omega$ such that $d(\log \Omega)=\psi$. We define $\Omega_{i}=d \Omega\left(e_{i}\right)$ and 
$W=\Omega / h$. Then $d h\left(e_{i}\right)=\Omega_{i}\left(1+\Omega \lambda^{i} / W\right) / W$ and (17) holds. Moreover, it follows from (4) that (16) and (18) are satisfied. Conversely if (16)-(18) hold, considering $Z^{i}=\Omega_{i} / W$ one concludes that (4) is satisfied. We define $h=\Omega / W$, then it follows that $d h\left(e_{i}\right)=Z^{i}\left(1+h \lambda^{i}\right)$.

We observe that it follows from the proof of Proposition 1.2 that

$$
d h\left(e_{i}\right)=\frac{\Omega_{i}}{W}\left(1+\Omega \lambda^{i} / W\right) \quad Z^{i}=\frac{\Omega_{i}}{W} \quad \Delta=\frac{\sum_{j}\left(\Omega_{j}\right)^{2}}{(W)^{2}} .
$$

Hence $d h\left(e_{i}\right) \neq 0$ if and only if $\Omega_{i} \neq 0$. For each solution $\Omega_{i}, 1 \leq i \leq 2$, of (16), there exists a 2-parameter family of solutions of the system (17), (18). In fact, Equation (16) is the integrability condition of the system of equations (17), (18) for $\Omega$ and $W$.

The Ribaucour transformation of a surface is given in terms of the solutions of the above system.

Theorem 1.3. Let $M$ be an orientable surface of $R^{3}$, without umbilic points, parametrized by $X: U \subset R^{2} \rightarrow M$. Assume $e_{i}, 1 \leq i \leq 2$ are orthogonal principal directions, $\lambda^{i}$ the corresponding principal curvatures and $N$ is a unit vector field normal to $M$. A surface $\widetilde{M}$ is locally associated to $M$, by a Ribaucour transformation, if and only if, there exist differentiable functions $W, \Omega, \Omega_{i}: V \subset U \rightarrow R$, which satisfy (16)-(18), such that $W\left(W+\Omega \lambda^{i}\right)\left(\Omega_{i} S-\right.$ $\left.\Omega d S\left(e_{i}\right)\right) \neq 0, \forall i$ and $\widetilde{X}: V \subset R^{2} \rightarrow \widetilde{M}$, is a parametrization of $\widetilde{M}$ given by

$$
\tilde{X}=X-\frac{2 \Omega}{S}\left(\sum_{i} \Omega_{i} e_{i}-W N\right),
$$

where

$$
S=\sum_{i}\left(\Omega_{i}\right)^{2}+W^{2} .
$$

Moreover, the normal map of $\widetilde{X}$ is given by

$$
\widetilde{N}=N+\frac{2 W}{S}\left(\sum_{i} \Omega_{i} e_{i}-W N\right)
$$

and the principal curvatures of $\widetilde{X}$ for each $1 \leq i \leq 2$ are given by

$$
\tilde{\lambda}^{i}=\frac{d S\left(e_{i}\right) W+\Omega_{i} \lambda^{i} S}{\Omega_{i} S-\Omega d S\left(e_{i}\right)} \quad \text { if } \Omega_{i} \neq 0 .
$$

Proof. Let $X$ and $\widetilde{X}$ be parametrizations of $M$ and $\widetilde{M}$. We have seen in Theorem 1.1 that the normal vector field $\widetilde{N}$ is given by (2). Hence it follows from (19) and (9) that (22) holds. The expression (20) follows directly from (1) and (22). The condition $W\left(W+\Omega \lambda^{i}\right) \neq 0, \forall i$ follows from the fact that $h=\Omega / W$ and $1+h \lambda^{i} \neq 0$. 
If $\Omega_{i} \neq 0$, then we consider (15). It follows from (7)-(9) and (19) that

$$
L_{i}^{3}=2 \frac{W}{S^{2}}\left(W d S\left(e_{i}\right)+\Omega_{i} S \lambda^{i}\right) .
$$

Therefore, using (19) and (9) we have that

$$
d h\left(e_{i}\right)\left(1-b^{3}\right)-h L_{i}^{3}=2 \frac{W}{S^{2}}\left(\Omega_{i} S-\Omega d S\left(e_{i}\right)\right) .
$$

From the last two relations we conclude that (23) holds.

We observe that, eventually, the parametrization of $\widetilde{M}$ given by (20) may extend regularly to wherever $W\left(W+\Omega \lambda^{i}\right)$ vanishes (see for example Section 2). From now on, whenever we say that a surface $\widetilde{M}$ is locally associated by a Ribaucour to a surface $M$, we are assuming that there are functions $\Omega, \Omega_{i}$ and $W$ locally defined, satisfying (16)-(18).

We now provide a sufficient condition for a Ribaucour transformation to transform a linear Weingarten surface into another such surface.

Theorem 1.4. Let $M$ be a surfaces of $R^{3}$, without umbilic points and let $\widetilde{M}$ be associated to $M$ by a Ribaucour transformation, such that the normal lines intersect at a distance function $h$. Assume that $h=\Omega / W$ is not constant along the lines of curvature and the functions $\Omega_{i}, \Omega$ and $W$ satisfy the additional relation

$$
S=2 c\left(\alpha \Omega^{2}+\beta \Omega W+\gamma W^{2}\right),
$$

where $S$ is defined by (21), $c \neq 0$ and $\alpha, \beta, \gamma$ are real constants. Then $\widetilde{M}$ is a linear Weingarten satisfying $\alpha+\beta \widetilde{H}+\gamma \widetilde{K}=0$, if and only if $\alpha+\beta H+\gamma K=0$ holds for the surface $M$, where $K, H$ and $\widetilde{K}, \widetilde{H}$ are the Gaussian and mean curvatures of $M$ and $\widetilde{M}$ respectively. Moreover, $\widetilde{M}$ has no umbilic points.

Proof. We will introduce the following notation for the right-hand side of the algebraic equation (24):

$$
P=\alpha \Omega^{2}+\beta \Omega W+\gamma W^{2} .
$$

Since $S$ satisfies $S=2 c P$, it follows from (17) and (18) that

$$
d S=2 c d P=2 c \sum_{i}\left[(2 \alpha \Omega+\beta W) \omega_{i}+(2 \gamma W+\beta \Omega) \omega_{i 3}\right] \Omega_{i} .
$$

Therefore,

$$
\begin{aligned}
W d S\left(e_{i}\right)+S \Omega_{i} \lambda^{i} & =2 c \Omega_{i}\left\{\left[(2 \alpha \Omega+\beta W)-(2 \gamma W+\beta \Omega) \lambda^{i}\right] W+\lambda^{i} P\right\} \\
S \Omega_{i}-\Omega d S\left(e_{i}\right) & =2 c \Omega_{i}\left(P-\Omega\left[(2 \alpha \Omega+\beta W)-(2 \gamma W+\beta \Omega) \lambda^{i}\right]\right) .
\end{aligned}
$$


By assumption $d h\left(e_{i}\right) \neq 0$, i.e., $\Omega_{i} \neq 0$, for all $i$, therefore from (23), and (27) we get

$$
\tilde{\lambda}^{i}=\frac{2 \alpha \Omega W+\beta W^{2}+\lambda^{i}\left(\alpha \Omega^{2}-\gamma W^{2}\right)}{\left(2 \gamma \Omega W+\beta \Omega^{2}\right) \lambda^{i}-\left(\alpha \Omega^{2}-\gamma W^{2}\right)}
$$

In order to conclude the proof we introduce the following notation:

$$
L=2 \alpha \Omega W+\beta W^{2} \quad T=\alpha \Omega^{2}-\gamma W^{2} \quad Q=2 \gamma \Omega W+\beta \Omega^{2} .
$$

Then, $\widetilde{\lambda}^{i}=\left(L+\lambda^{i} T\right) /\left(Q \lambda^{i}-T\right)$ and hence, the numerator of $\alpha-\frac{\beta}{2}\left(\widetilde{\lambda}^{1}+\right.$ $\left.\widetilde{\lambda}^{2}\right)+\gamma \widetilde{\lambda}^{1} \widetilde{\lambda}^{2}$ is equal to

$$
\begin{aligned}
\alpha T^{2}+\beta L T+\gamma L^{2}+H\left(2 \alpha T Q-\beta T^{2}+\beta L Q\right. & -2 \gamma L T) \\
& +K\left(\alpha Q^{2}-\beta T Q+\gamma T^{2}\right) .
\end{aligned}
$$

By substituting $\alpha, \beta H$ and $\gamma K$, on the right-hand side of this last equality, by the two other terms of the expression $\alpha+\beta H+\gamma K=0$, we get

$$
\alpha+\beta \widetilde{H}+\gamma \widetilde{K}=0 \text { if and only if }(\beta T+\gamma L-\alpha Q)(L-2 T H-Q K)=0,
$$

where the last equality follows from the fact that the expression $\beta T+\gamma L-\alpha Q$ is identically zero.

We conclude the proof of the theorem by observing that

$$
\widetilde{\lambda}^{2}-\widetilde{\lambda}^{1}=\frac{L Q+T^{2}}{\left(Q \lambda^{1}-T\right)\left(Q \lambda^{2}-T\right)}\left(\lambda^{2}-\lambda^{1}\right) .
$$

Since $L Q+T^{2}=P^{2}=S^{2} /\left(4 c^{2}\right) \neq 0$ and $M$ has no umbilic points, it follows that $\widetilde{M}$ has no umbilic points.

The natural question one poses is if the system (16)-(18) with the additional condition (24) is integrable, whenever we start with a linear Weingarten surface. The following theorem answers this question affirmatively:

Theorem 1.5. Let $M$ be a surface of $R^{3}$, which satisfies $\alpha+\beta H+\gamma K=$ 0 and $H^{2}-K>0$. Then the system of equations (16)-(18) and (24) is integrable and the solution is uniquely determined on a simply connected domain $U$ by any given initial condition satisfying (24). Moreover, whenever $\alpha \neq 0$, any solution of the system defined on $U$ is either identically zero and hence anihilates $S$ or else the function $S$ does not vanish on $U$. 
Proof. We initially observe that as a consequence of (16)-(18) and (24) we only need to prove that the system

$$
\begin{aligned}
d \Omega & =\sum_{i} \Omega_{i} \omega_{i} \\
d W & =\sum_{i} \Omega_{i} \omega_{i 3} \\
d \Omega_{i} & =\Omega_{j} \omega_{i j}+c(2 \alpha \Omega+\beta W) \omega_{i}-[(1-2 c \gamma) W-c \beta \Omega] \omega_{i 3} \quad i \neq j
\end{aligned}
$$

is integrable.

We start by showing that if (29) holds then $S-2 c P$ is a constant function, where $S$ and $P$ are defined by (21) and (25) respectively. In fact,

$$
\begin{aligned}
d S-2 c d P & =2 \sum_{i} \Omega_{i} d \Omega_{i}+2(W-c \beta \Omega-2 c \gamma W) d W-2 c(2 \alpha \Omega+\beta W) d \Omega \\
& =2 \sum_{i, j} \Omega_{i} \Omega_{j} \omega_{i j}=0 .
\end{aligned}
$$

Therefore, by choosing the initial condition at a point such that $S=2 c P$, we will have (24) identically satisfied on a connected domain.

Now we consider the ideal $\mathcal{I}$ generated by the 1 -forms

$$
\begin{aligned}
\theta & =d \Omega-\sum_{i} \Omega_{i} \omega_{i} \\
\varphi & =d W-\sum_{i} \Omega_{i} \omega_{i 3} \\
\theta_{i} & =d \Omega_{i}-\Omega_{j} \omega_{i j}-c(2 \alpha \Omega+\beta W) \omega_{i}+[(1-2 c \gamma) W-c \beta \Omega] \omega_{i 3} \quad i \neq j .
\end{aligned}
$$

A straightforward computation shows that $d \theta=-\sum_{i} \theta_{i} \wedge \omega_{i}$ and $d \varphi=$ $-\sum_{i} \theta_{i} \wedge \omega_{i 3}$. Similarly, using (30) we obtain that

$$
\begin{aligned}
d \theta_{i}= & -\theta_{j} \wedge \omega_{i j}+\varphi \wedge\left[(1-2 c \gamma) \omega_{i 3}-c \beta \omega_{i}\right]-c \theta \wedge\left(\beta \omega_{i 3}+2 \alpha \omega_{i}\right)+ \\
& +2 c \Omega_{j}(\alpha+\beta H+\gamma K) \omega_{i} \wedge \omega_{j}
\end{aligned}
$$

where $i \neq j$. Since the surface is linear Weingarten, it follows that $\mathcal{I}$ is closed under exterior differentiation, hence the system (29) is integrable.

Assume that $S\left(p_{0}\right)=0$ for $p_{0} \in U$. Then it follows from (21) that $\Omega_{1}$, $\Omega_{2}$ and $W$ vanish at $p_{0}$. Since (24) holds we conclude that if $\alpha \neq 0$, then $\Omega\left(p_{0}\right)=0$. Since $U$ is simply connected, the uniqueness of solutions for the system implies that $\Omega \equiv \Omega_{1} \equiv \Omega_{2} \equiv W \equiv 0$ and hence $S \equiv 0$. This concludes the proof of the theorem.

As a consequence of Theorems 1.3 and 1.4 we obtain:

Theorem 1.6. Let $M$ be a linear Weingarten surface, without umbilic points, satisfying $\alpha+\beta H+\gamma K=0$ and locally parametrized by $X: U \subset$ 
$R^{2} \rightarrow M \subset R^{3}$. Any linear Weingarten parametrized surface, locally associated to $X$ by a Ribaucour transformation as in Theorem 1.5 is given by

$$
\tilde{X}=X-\frac{2 \Omega}{S}\left(\sum_{i} \Omega_{i} e_{i}-W N\right),
$$

where $e_{i}$ are orthogonal principal directions, $\Omega, \Omega_{i}, W$ are solutions of (16)(18) and (24), and $\widetilde{X}$ is an immersed surface defined on

$$
\widetilde{U}=\left\{\left(u_{1}, u_{2}\right) \in U ; T^{2}+2 T Q H+Q^{2} K \neq 0\right\}
$$

where $T=\alpha \Omega^{2}-\gamma W^{2}$ and $Q=2 \gamma \Omega W+\beta \Omega^{2}$.

Proof. We only need to show that $\tilde{X}$, defined by (31), is a parametrized surface of $R^{3}$. With the same notation introduced in the proof of Theorem 1.4, we observe that $S=2 c P$, where $P$ is defined by (25) and the differential of $S$ is given by (26). Therefore, we get that

$$
d\left(\frac{\Omega}{S}\right)=\frac{1}{2 c P^{2}} \sum_{k} \Omega_{k} \eta_{k}
$$

where $\eta_{k}$ for $k=1,2$ is a 1 -form defined by

$$
\eta_{k}=\left(\gamma W^{2}-\alpha \Omega^{2}\right) \omega_{k}-\left(\beta \Omega^{2}+2 \gamma \Omega W\right) \omega_{k 3} .
$$

It follows from this expression and the last two equations of system (29) that

$$
d \tilde{X}=\frac{1}{P} \sum_{i=1}^{2} \eta_{i} \widetilde{e}_{i}
$$

where

$$
\begin{aligned}
& \widetilde{e}_{1}=\frac{1}{c P}\left[\left(c P-\Omega_{1}^{2}\right) e_{1}-\Omega_{1} \Omega_{2} e_{2}+W \Omega_{1} e_{3}\right] \\
& \widetilde{e}_{2}=\frac{1}{c P}\left[-\Omega_{1} \Omega_{2} e_{1}+\left(c P-\Omega_{2}^{2}\right) e_{2}+W \Omega_{2} e_{3}\right] .
\end{aligned}
$$

A simple computation shows that these vectors are orthonormal. Therefore, $\widetilde{X}$ is an immersion wherever $\eta_{1} \wedge \eta_{2} \neq 0$, i.e., on the subset $\widetilde{U}$ described by $(32)$.

Remark 1.7. We observe that, as a consequence of the proof of Theorem 1.6, the principal directions $\widetilde{e}_{1}, \widetilde{e}_{2}$, of $\widetilde{X}$ are given by (35) and (36). Moreover, its dual forms, which are determined by (34), are given by $\widetilde{\omega}_{i}=$ $\frac{1}{P}\left(\gamma W^{2}-\alpha \Omega^{2}+(\beta \Omega+2 \gamma W) \Omega \lambda^{i}\right) \omega_{i}$. We also observe that the surfaces described in Theorem 1.6 depend on 4 parameters. However, in some cases the number of parameters may reduce to one (the parameter $c$ ), if we exclude surfaces which are congruent by rigid motions of $R^{3}$. 
Our next results give the $\mathrm{H}-\mathrm{cmc}$ case, which is obtained by considering $H$ a nonzero constant, $\alpha=-H, \beta=1$ and $\gamma=0$ on Theorems 1.4-1.6. For later use, we will explicitly give the corresponding results.

Corollary 1.8. Let $M$ be a regular surface of $R^{3}$, with no umbilic point. Let $\widetilde{M}$ be associated by a Ribaucour transformation to $M$, such that the normal lines intersect at a distance function $h$. Assume that $h=\Omega / W$ is not constant along the lines of curvature and the functions $\Omega_{i}, \Omega$ and $W$ satisfy the additional relation

$$
S=2 c \Omega(-H \Omega+W),
$$

where $S$ is defined by (21), $c \neq 0$ and $H \neq 0$ are real constants. Then $\widetilde{M}$ is an $H$-cmc surface, if and only if, $M$ is an $H$-cmc surface. Moreover, $\widetilde{M}$ has no umbilic points.

Corollary 1.9. Let $M$ be an $H$-cmc surface of $R^{3}$. Then the system of Equations (16)-(18) and (37) is integrable and the solution is uniquely determined on a simply connected domain $U$ by any given initial condition satisfying (37). Moreover, for any solution of the system defined on $U$ the function $S$ does not vanish on $U$.

As a consequence of Theorem 1.6, we get our next result. We observe that in the case of $H$-cmc surfaces, by considering $\alpha=-H, \beta=1$ and $\gamma=0$, we conclude that (32) reduces to $\Omega^{4}\left(K-H^{2}\right) \neq 0$.

Corollary 1.10. Let $M$ be an $H$-cmc surface with no umbilic points, locally parametrized by $X: U \subset R^{2} \rightarrow M \subset R^{3}$. Any $H$-cmc parametrized surface, locally associated to $X$ by a Ribaucour transformation as in Corollary 1.9 is given by

$$
\widetilde{X}=X-\frac{1}{c(W-H \Omega)}\left(\sum_{i} \Omega_{i} e_{i}-W N\right),
$$

where $e_{i}$ are orthogonal principal directions, $\Omega, \Omega_{i}, W$ are solutions of (16)(18) and (37) and the constant $c$ satisfies $c(c-2 H)>0$.

Proof. We only need to prove that $c$ satisfies $c(c-2 H)>0$. This follows from the algebraic condition (37), which can be written as

$$
\sum_{i}\left(\Omega_{i}\right)^{2}+(W-c \Omega)^{2}-c(c-2 H) \Omega^{2}=0 .
$$

Remark 1.11. It follows from the proof of Theorem 1.6 that if $M$ and $\widetilde{M}$ are $H$-cmc surfaces associated by a Ribaucour transformation as in Corollary 1.10, then the principal directions $\widetilde{e}_{1}, \widetilde{e}_{2}$, of $\widetilde{M}$ are given by (35) and 
(36) where $P=\Omega(W-H \Omega)$. Its dual forms are,

$$
\widetilde{\omega}_{i}=\frac{\left(H+\lambda^{i}\right) \Omega}{W-H \Omega} \omega_{i} .
$$

Moreover, it follows from (28) that

$$
\tilde{\lambda}_{i}=\frac{W^{2}-H \Omega\left(2 W+\lambda^{i} \Omega\right)}{\Omega^{2}\left(\lambda^{i}+H\right)}
$$

and the Gaussian curvature is given by

$$
\widetilde{K}=H^{2}+\frac{(W-H \Omega)^{4}}{\Omega^{4}\left(K-H^{2}\right)} .
$$

\section{Families of linear Weingarten surfaces associated to the cylinder.}

In this section, by applying Theorem 1.6 to the cylinder, we obtain a two parameter $(c, \gamma)$ family of complete linear Weingarten surfaces. The parameters belong to a region composed by two connected components of $R^{2}$. One of these components contains curves which provide $n$-bubble surfaces (Weingarten and $\mathrm{cmc}$ ) which are 1-periodic, have genus zero and two ends of finite geometric index. We also show that their total curvature vanishes, while the total absolute curvature is $8 \pi n$.

Proposition 2.1. Consider the cylinder parametrized by

$$
X\left(u_{1}, u_{2}\right)=\left(\cos \left(u_{2}\right), \sin \left(u_{2}\right), u_{1}\right) \quad\left(u_{1}, u_{2}\right) \in R^{2}
$$

as a linear Weingarten surface satisfying $-1 / 2+H+\gamma K=0$. A parametrized surface is a linear Weingarten surface locally associated to $X$ by a Ribaucour transformation as in Theorem 1.5, if and only if, it is given by

$$
\widetilde{X}_{c \gamma}=X-\frac{2(f+g)}{c\left[(2 \gamma+1) g^{2}-f^{2}\right]}\left(f^{\prime} X_{u_{1}}+g^{\prime} X_{u_{2}}-g N\right)
$$

where $N$ is the inner unit normal vector field of the cylinder, $c \neq 0$ and $\gamma$ are real constants such that

$$
\xi(c, \gamma)=1-c(2 \gamma+1)
$$

and $c$ are not simultaneously positive, and $f\left(u_{1}\right), g\left(u_{2}\right)$ are solutions of the equations

$$
\begin{aligned}
& f^{\prime \prime}+c f=0, \\
& g^{\prime \prime}+\xi g=0
\end{aligned}
$$

with initial conditions satisfying

$$
\left(\left(f^{\prime}\right)^{2}+\left(g^{\prime}\right)^{2}+\xi g^{2}+c f^{2}\right)\left(u_{1}^{0}, u_{2}^{0}\right)=0 .
$$


Moreover, $\widetilde{X}_{c \gamma}$ is a regular surface defined on the subset of $U$ where

$$
\left((f+g)^{2}+2 \gamma g^{2}\right)\left(f^{2}+2(2 \gamma+1) f g+(2 \gamma+1) g^{2}\right) \neq 0 .
$$

Proof. The first fundamental form of the cylinder is given by $d s^{2}=d u_{1}^{2}+d u_{2}^{2}$ and $\lambda^{1}=0, \lambda^{2}=-1$. In order to obtain the Ribaucour transformations, we need to solve the following system of equations, which is obtained from (16)-(18):

$$
\frac{\partial \Omega_{i}}{\partial u_{j}}=0, \quad \frac{\partial \Omega}{\partial u_{i}}=\Omega_{i}, \quad \frac{\partial W}{\partial u_{i}}=-\Omega_{i} \lambda^{i}, \quad 1 \leq i \neq j \leq 2 .
$$

The associated surface will be linear Weingarten when $\Omega_{1}$ and $\Omega_{2}$ satisfy $\partial \Omega_{1} / \partial u_{1}=c(W-\Omega)$ and $\partial \Omega_{2} / \partial u_{2}=(c-1+2 c \gamma) W$.

Since $\Omega_{u_{1} u_{2}}=0$, it follows that $\Omega=f\left(u_{1}\right)+g\left(u_{2}\right)$, where $f$ and $g$ are functions of $u_{1}$ and $u_{2}$ respectively. Therefore $\Omega_{1}=f^{\prime}$ and $\Omega_{2}=g^{\prime}$. Moreover, $W=g+a$, where $a$ is a real constant and the functions $f$ and $g$ satisfy the following equations:

$$
\begin{aligned}
f^{\prime \prime}+c f-c a & =0 \\
g^{\prime \prime}+\xi(g+a) & =0 .
\end{aligned}
$$

It follows from these equations and the expressions of $\Omega$ and $W$, that without loss of generality we can consider $a=0$. Therefore, $f$ and $g$ must satisfy Equations (45) and (46) and the algebraic condition (24), which reduces to (47). Moreover, since this last condition should be identically satisfied by the nontrivial solution functions $f$ and $g$, we conclude that the constants $c \neq 0$ and $\gamma$ are such that $c$ and $\xi$ cannot be simultaneously positive.

Moreover, from (20) we conclude that the associated linear Weingarten surface is given by (43). From (32) we obtain the domain where $\widetilde{X}$ is regular, which is described by (48).

The family of linear Weingarten surfaces given by (43) includes the cylinder. In fact, if we choose the initial conditions such that $f \equiv 0, g \neq 0$ for $\xi \leq 0$ or $f \neq 0, g \equiv 0$ for $c<0$, we get a reparametrization of the cylinder.

Each linear Weingarten surface associated to the cylinder as in Proposition 2.1, is parametrized by lines of curvature and the metric is given (see Remark 1.7) by $d s^{2}=\psi_{1}^{2} d u_{1}^{2}+\psi_{2}^{2} d u_{2}^{2}$, where

$$
\psi_{1}=\frac{(f+g)^{2}+2 \gamma g^{2}}{(1+2 \gamma) g^{2}-f^{2}} \quad \text { and } \quad \psi_{2}=\frac{(1+2 \gamma) g^{2}+2(1+2 \gamma) f g+f^{2}}{(1+2 \gamma) g^{2}-f^{2}}
$$

These expressions show that the Ribaucour transformation, applied to the cylinder for $\gamma \neq 0$, is not a Darboux transformation. 
We now introduce a notation, which will be useful in the following results. A rotation of angle $\theta$ in the $x y$ plane of $R^{3}$ will be denoted by

$$
R_{\theta}=\left(\begin{array}{ccc}
\cos \theta & -\sin \theta & 0 \\
\sin \theta & \cos \theta & 0 \\
0 & 0 & 1
\end{array}\right) .
$$

We denote by $T_{\delta}$ the translation defined by

$$
T_{\delta}(x, y, z)=(x, y, z+\delta) .
$$

Proposition 2.2. Consider the linear Weingarten surfaces associated to the cylinder and parametrized by (43). Excluding the cylinder:

i) If $c \xi \geq 0$, then any surface $\widetilde{X}_{c \gamma}$ has curves of singularities.

ii) If $c \xi<0$ then, up to rigid motions of $R^{3}$, the surface $\widetilde{X}_{c \gamma}$ is determined by the functions

$$
\begin{array}{llrl}
f=\varepsilon_{1} \sqrt{|\xi|} \sin \left(\sqrt{c} u_{1}\right) & g=\varepsilon_{2} \sqrt{c} \cosh \left(\sqrt{|\xi|} u_{2}\right) & \text { if } c>0, \xi<0 \\
f=\varepsilon_{1} \sqrt{\xi} \cosh \left(\sqrt{|c|} u_{1}\right) & g=\varepsilon_{2} \sqrt{|c|} \sin \left(\sqrt{\xi} u_{2}\right) & \text { if } c<0, \xi>0
\end{array}
$$

where $\varepsilon_{i}= \pm 1, c \neq 0$ and $\gamma$ are real numbers and $\xi(c, \gamma)$ is defined by (44).

Proof. We observe that the functions $f$ and $g$ of the family of surfaces described by (43) are given by

$$
\begin{aligned}
& f= \begin{cases}a_{1} \cos \left(\sqrt{c} u_{1}\right)+b_{1} \sin \left(\sqrt{c} u_{1}\right) & \text { if } \quad c>0, \\
a_{1} \cosh \left(\sqrt{|c|} u_{1}\right)+b_{1} \sinh \left(\sqrt{|c|} u_{1}\right) & \text { if } \quad c<0,\end{cases} \\
& g=\left\{\begin{array}{lll}
a_{2} u_{2}+b_{2} & \text { if } \quad \xi=0, \\
a_{2} \cosh \left(\sqrt{|\xi|} u_{2}\right)+b_{2} \sinh \left(\sqrt{|\xi|} u_{2}\right) & \text { if } \quad \xi<0, \\
a_{2} \cos \left(\sqrt{\xi} u_{2}\right)+b_{2} \sin \left(\sqrt{\xi} u_{2}\right) & \text { if } \quad \xi>0,
\end{array}\right.
\end{aligned}
$$

and the constants satisfy the algebraic relation given by (47),

$$
\begin{array}{ll}
a_{1}=b_{1}=a_{2}=0 & \text { if } \quad c>0 \text { and } \xi=0, \\
c\left(b_{1}^{2}-a_{1}^{2}\right)-a_{2}^{2}=0 & \text { if } \quad c<0 \text { and } \xi=0, \\
c\left(b_{1}^{2}+a_{1}^{2}\right)+\xi\left(a_{2}^{2}-b_{2}^{2}\right)=0 & \text { if } \quad c>0 \text { and } \xi<0, \\
c\left(b_{1}^{2}-a_{1}^{2}\right)-\xi\left(a_{2}^{2}-b_{2}^{2}\right)=0 & \text { if } \quad c<0 \text { and } \xi<0, \\
c\left(b_{1}^{2}-a_{1}^{2}\right)-\xi\left(a_{2}^{2}+b_{2}^{2}\right)=0 & \text { if } \quad c<0 \text { and } \xi>0 .
\end{array}
$$

If $c>0$, and $\xi=0$, then the surface $\widetilde{X}$ reduces to the cylinder. If $c<0$ and $\xi=0$, there are curves in $R^{2}$ where (48) vanishes.

If $c \xi>0$, since by Proposition $2.1 c$ and $\xi(c, \gamma)$ cannot be simultaneously positive, then we may only have $c<0$ and $\xi<0$. In this case, the functions $f$ and $g$ are defined as above, $\gamma<-1 / 2$ and there are four curves on $R^{2}$ determined by (48)

$$
f+g \pm \sqrt{2|\gamma|} g=0 \quad f+(2 \gamma+1) g \pm \sqrt{2 \gamma(2 \gamma+1)} g=0
$$


where $\tilde{X}$ is not regular.

If $c>0$ and $\xi<0$, then by choosing $a_{1}=b_{1}=0$ we have $b_{2}= \pm a_{2}, f=0$, $g=a_{2} \exp \left( \pm \sqrt{|\xi|} u_{2}\right)$ and the surface $\tilde{X}$ reduces to a reparametrization of the cylinder. Therefore, excluding the cylinder, we may assume $a_{1}^{2}+b_{1}^{2} \neq 0$ and

$$
f=\varepsilon_{1} \sqrt{|\xi|} \sin \left(A+\sqrt{c} u_{1}\right) \quad g=\varepsilon_{2} \sqrt{c} \cosh \left(B+\sqrt{|\xi|} u_{2}\right) .
$$

Similarly, If $c<0$ and $\xi>0$, then excluding the cylinder, we may assume $a_{2}^{2}+b_{2}^{2} \neq 0$ and hence

$$
f=\varepsilon_{1} \sqrt{\xi} \cosh \left(A+\sqrt{|c|} u_{1}\right) \quad g=\varepsilon_{2} \sqrt{|c|} \sin \left(B+\sqrt{\xi} u_{2}\right) .
$$

We conclude the proof by observing that the constants $A$ and $B$, without loss of generality, may be considered to be zero. One can verify that the surfaces with different values of $A, B$ are congruent by rigid motions of $R^{3}$. In fact, using the notation $\widetilde{X}_{c \gamma A B}$ for the surface $\widetilde{X}_{c \gamma}$ with fixed constants $A$ and $B$, we have

$$
\widetilde{X}_{c \gamma A B}=R_{-\frac{B}{\sqrt{|\xi|}}} \widetilde{X}_{c \gamma 00} \circ h+T_{-\frac{A}{\sqrt{|c|}}}
$$

where $h\left(u_{1}, u_{2}\right)=\left(u_{1}+A / \sqrt{|c|}, u_{2}+B / \sqrt{|\xi|}\right)$.

We observe that it follows from the above expressions that the function $P$ defined by $(25)$, reduces to

$$
P=\left[(2 \gamma+1) g^{2}-f^{2}\right] / 2
$$

and it does not vanish on $R^{2}$. This will be useful for considering global properties of the surfaces obtained in Proposition 2.2.

In order to study the regularity of the surfaces obtained in Proposition 2.2, we introduce the following notation:

$$
\begin{aligned}
& h_{1}(c, \gamma)=2 c(2 \gamma+1)(\sqrt{2 \gamma(2 \gamma+1)}-2 \gamma)-1, \\
& h_{2}(c, \gamma)=2 c(\sqrt{2|\gamma|}+2 \gamma)-1, \\
& h_{3}(c, \gamma)=-2 c(2 \gamma+1)(\sqrt{2 \gamma(2 \gamma+1)}+2 \gamma)-1, \\
& h_{4}(c, \gamma)=2 c(\sqrt{2|\gamma|}-2 \gamma)+1, \\
& h_{5}(c, \gamma)=-2 c(2 \gamma+1)(\sqrt{2 \gamma(2 \gamma+1)}-2 \gamma)+1 .
\end{aligned}
$$

Our next result shows that a surface $\tilde{X}_{c \gamma}$ given by (43) is an immersion of $R^{2}$ if and only if the pair $(c, \gamma)$ belongs to a region with two connected components of $R^{2}$ determined by the functions $\xi(c, \gamma)$ and $h_{1}(c, \gamma) \ldots h_{5}(c, \gamma)$ (see Figure 1). 


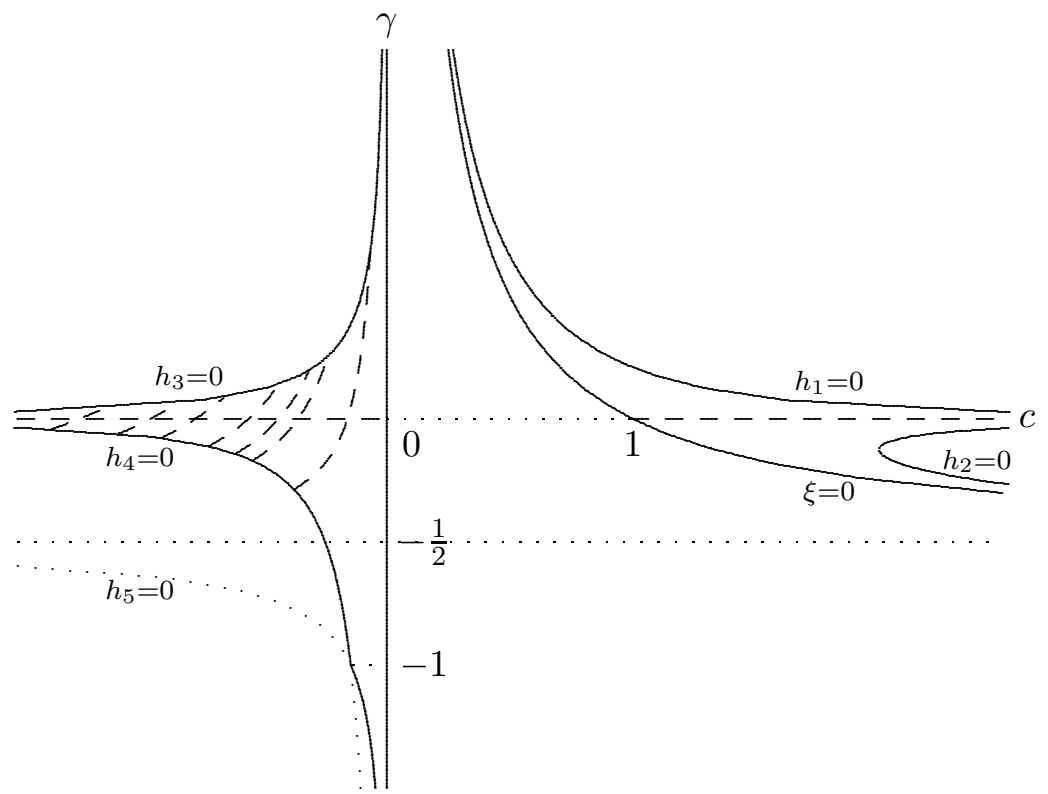

Figure 1. Any pair $(c, \gamma)$, in each of the two connected components, generates a complete linear Weingarten surface, which satisfies the relation $-1 / 2+H-\gamma K=0$ and it is cmc when $\gamma=0$. The dashed curves in the left region, given by $1-c(2 \gamma+1)=n^{2} / m^{2}$, generate 1-periodic $n$-bubble surfaces with two ends of geometric index $m$ (see Figure 2).

Proposition 2.3. A Weingarten surface $\widetilde{X}_{c \gamma}$ given by Proposition 2.2 is an immersion of $R^{2}$, if and only if, $c \xi(c, \gamma)<0$ and the pair $(c, \gamma)$ belongs to one of the following subsets of $R^{2}$ :

i) $c>0$ and one of the following holds:

a) $\gamma \geq 0$ and $\xi(c, \gamma)<0, h_{1}(c, \gamma)<0$;

b) $-1 / 2<\gamma<0$ and $\xi(c, \gamma)<0, h_{2}(c, \gamma)<0$.

ii) $c<0$ and one of the following holds:

a) $\gamma \geq 0$ and $h_{3}(c, \gamma)<0$

b) $-1 \leq \gamma<0$ and $h_{4}(c, \gamma)>0$;

c) $\gamma \leq-1$ and $h_{5}(c, \gamma)>0$.

Proof. From Proposition 2.2, we have seen that if the surface $\widetilde{X}$ is a regular immersion of $R^{2}$, then excluding the cylinder, we only need to consider $c \xi<0$. 
i) If $c>0$ and $\xi<0$ then the functions $f$ and $g$ are given by (52). If $\gamma \geq 0, \widetilde{X}_{c \gamma}$ is an immersion of $R^{2}$ if and only if the second factor of (48) does not vanish. It follows from (44) that this is equivalent to having $h_{1}(c, \gamma)<0$, where $h_{1}$ is defined by $(55)$.

If $\gamma<0$, then $1 / c-1<2 \gamma<0$. Hence, $c>1$ and $-1 / 2<\gamma<0$. Then $\widetilde{X}_{c \gamma}$ is a regular immersion of $R^{2}$, if and only if the first factor of (48) does not vanish i.e., $1 /(2 \gamma+1)<c<1 / 2(\sqrt{2|\gamma|}+2 \gamma)$, or equivalently $h_{2}(c, \gamma)<0$, where $h_{2}$ is defined by (56). This concludes the proof of i).

ii) If $c<0$ and $\xi(c, \gamma)<0$, then the surface $\widetilde{X}_{c \gamma}$ is defined by (43) and the functions $f$ and $g$ are given by (53). We need to consider four cases for $\gamma$. If $\gamma \geq 0$, then the nonvanishing of the second factor of (48) is equivalent to $\sqrt{|c|}(2 \gamma+1+\sqrt{2 \gamma(2 \gamma+1)})<\sqrt{\xi}$, i.e., $h_{3}(c, \gamma)<0$, where $h_{3}$ is defined by (57). If $-1 / 2<\gamma<0$, from the first factor of (48) we conclude that the pair $(c, \gamma)$ must satisfy $h_{4}(c, \gamma)>0$, where $h_{4}$ is defined by (58).

If $\gamma \leq-1 / 2$, then both factors of (48) should not vanish on $R^{2}$ This occurs if and only if the following inequalities hold:

$$
|1 \pm \sqrt{2|\gamma|}| \sqrt{|c|}<\sqrt{\xi} \quad|2 \gamma+1 \pm \sqrt{2 \gamma(2 \gamma+1)}| \sqrt{|c|}<\sqrt{\xi} .
$$

If $-1 \leq \gamma \leq-1 / 2$ then $\sqrt{2 \gamma(2 \gamma+1)}-2 \gamma-1 \leq 1+\sqrt{2|\gamma|}$. Hence, the system (60) holds if and only if $h_{4}(c, \gamma)>0$. If $\gamma \leq-1$ then $1+\sqrt{2|\gamma|} \leq$ $\sqrt{2 \gamma(2 \gamma+1)}-2 \gamma-1$. Therefore, (60) holds if and only if

$$
(\sqrt{2 \gamma(2 \gamma+1)}-2 \gamma-1) \sqrt{|c|}<\sqrt{\xi}
$$

i.e., $h_{5}(c, \gamma)>0$, where $h_{5}$ is defined by (59). This concludes the proof.

Our next result shows that the linear Weingarten surfaces, locally associated to the cylinder by a Ribaucour transformation, are asymptotically close to cylinders.

Proposition 2.4. Let $X\left(u_{1}, u_{2}\right)$ be the parametrized cylinder given by (42). Any linear Weingarten surface $\widetilde{X}_{c \gamma}$ given by Proposition 2.3, satisfies the following:

i) If $c<0$ and $\xi(c, \gamma)>0$ then $\forall \varepsilon>0$ there exists $L>0$ such that

$$
\left|\widetilde{X}_{c \gamma}\left(u_{1}, u_{2}\right)-X\left(u_{1} \pm 2 / \sqrt{|c|}, u_{2}\right)\right|<\varepsilon \quad \forall\left(u_{1}, u_{2}\right) \in R^{2} \text { with } \pm u_{1} \geq L
$$

and

$$
\left|\frac{\partial^{i+j} \tilde{X}_{c \gamma}}{\partial u_{1}^{i} \partial u_{2}^{j}}\left(u_{1}, u_{2}\right)-\frac{\partial^{i+j} X}{\partial u_{1}^{i} \partial u_{2}^{j}}\left(u_{1}, u_{2}\right)\right|<\varepsilon,
$$

where $1 \leq i+j \leq 2$, and $i, j$ are nonnegative integer numbers. 
ii) If $c>0$ and $\xi(c, \gamma)<0$ then $\forall \varepsilon>0$ there exists $L>0$ such that $\left|\widetilde{X}_{c \gamma}\left(u_{1}, u_{2}\right)-X\left(u_{1}, u_{2} \pm \theta\right)\right|<\varepsilon \quad \forall\left(u_{1}, u_{2}\right) \in R^{2}$ with $\pm u_{2} \geq L$, where $\theta$ is such that $\cos \theta=1-2 /(c(1+2 \gamma))$ and $\sin \theta=-2 \sqrt{-\xi} /(c(1+$ $2 \gamma))$.

Proof. If $c<0$ and $\xi(c, \gamma)>0$ then the functions $f$ and $g$ are given by (53). It follows from a straightforward computation that

$$
\frac{c^{2}}{4}\left|\widetilde{X}_{c \gamma}\left(u_{1}, u_{2}\right)-X\left(u_{1} \pm 2 / \sqrt{|c|}, u_{2}\right)\right|^{2}=S_{1}^{2}+S_{2}^{2}+S_{3}^{2},
$$

where

$$
S_{1}=\frac{(f+g) f^{\prime}}{2 P} \pm \sqrt{|c|}, \quad S_{2}=\frac{(f+g) g^{\prime}}{2 P}, \quad S_{3}=\frac{(f+g) g}{2 P},
$$

where $P$ is given by (54). Since $g$ and $g^{\prime}$ are bounded functions, we have that $\lim _{u_{1} \rightarrow \pm \infty} S_{i}\left(u_{1}, u_{2}\right)=0$ uniformly with respect to $u_{2}$. Similarly, considering the difference of the first and second derivatives of $\widetilde{X}_{c \gamma}$ and $X$ as a linear combination of the vectors $X_{u_{1}}, X_{u_{2}}$ and $N$, one can show that each coefficient tends to 0 uniformly in $u_{2}$, when $u_{1} \rightarrow \pm \infty$. This concludes the proof of i). Similar arguments prove ii).

Our next result shows that the regular surfaces $\widetilde{X}_{c \gamma}$ given in Proposition 2.3 are complete. Moreover, the connected region described by ii) contains an infinite number of curves ( determined by considering $\sqrt{1-c(2 \gamma+1)}$ to be a rational number) such that the corresponding surfaces are 1-periodic $n$-bubbles whose total absolute curvature is $8 \pi n$.

Proposition 2.5. Any linear Weingarten surface $\widetilde{X}_{c \gamma}$, given by Proposition 2.3 is complete.

a) If $c<0$ and $\sqrt{\xi(c, \gamma)}=n / m$ is an irreducible rational number, then $\widetilde{X}_{c \gamma}$ is an immersion of a cylinder into $R^{3}$, with two ends of geometric index $m$ and $n$ isolated points of maximum (respectively minimum) for the Gaussian curvature. Moreover, the total curvature of $\widetilde{X}_{c \gamma}$ is zero, while its total absolute curvature is $8 \pi n$. The ends are embedded if and only if $m=1$. In this case they are cylindrical ends.

b) If $c>0$ or $c<0$ and $\sqrt{\xi}$ is not a rational number then $\widetilde{X}_{c \gamma}$ is an immersion of $R^{2}$ into $R^{3}$ (not periodic in any variable) with an infinite number of isolated critical points of its Gaussian curvature.

Proof. Assume $\xi>0$ and $c<0$ then the functions $f$ and $g$ are given by (53) and the coefficients of the first fundamental form $\psi_{i}$ of $\widetilde{X}_{c \gamma}$ are given by (49). Therefore, $\lim _{\left|u_{1}\right| \rightarrow \infty}\left|\psi_{i}\right|=1$ for $i=1,2$ uniformly in $u_{2}$. Hence, there 
exits $k>0$ such that $\left|\psi\left(u_{1}, u_{2}\right)\right|>1 / 2$ for all $\left(u_{1}, u_{2}\right) \in R^{2}$ with $\left|u_{1}\right|>k$. Let

$$
m_{i}=\min \left\{\left|\psi_{i}\left(u_{1}, u_{2}\right)\right|,\left(u_{1}, u_{2}\right) \in[-k, k] \times\left[0, \frac{2 \pi}{\sqrt{\xi}}\right]\right\} .
$$

Since $\widetilde{X}_{c \gamma}$ is regular, $m_{i}>0$. Moreover, $g\left(u_{2}\right)=g\left(u_{2}+2 \pi / \sqrt{\xi}\right)$, therefore $\left|\psi_{i}\left(u_{1}, u_{2}\right)\right| \geq m_{i}$ in $[-k, k] \times R$. Now consider $m_{0}=\min \left\{m_{1}, m_{2}, 1 / 2\right\}$, then $\left|\psi_{i}\right| \geq m_{0}$ in $R^{2}$. We conclude that $\widetilde{X}_{c \gamma}$ is a complete surface. The case $\xi<0$ and $c>0$ is analogous.

For $c<0$ if $\sqrt{\xi(c, \gamma)}=n / m$ is an irreducible rational number, then $\widetilde{X}_{c \gamma}$ is periodic in the variable $u_{2}$ with period $2 m \pi$. Hence it is an immersion of a cylinder into $R^{3}$. Moreover, the surface has two ends $\mathcal{F}^{ \pm}$corresponding to $u_{1} \rightarrow \pm \infty$.

It follows from (41) that the Gaussian curvature $\widetilde{K}$ of $\widetilde{X}_{c \gamma}$, is given by

$$
\widetilde{K}=\frac{2 f g\left(f^{2}+(1+2 \gamma) g^{2}\right)}{\left((f+g)^{2}+2 \gamma g^{2}\right)\left(f^{2}+(1+2 \gamma) g(2 f+g)\right)} .
$$

Therefore, the domain $R \times[0,2 m \pi]$ of $\widetilde{X}$ is composed of $2 n$ horizontal strips where $\widetilde{K}$ changes sign from positive to negative at each open consecutive strip and vanishes on the bordering straight lines. Moreover, $\lim _{u_{1} \rightarrow \pm \infty} \widetilde{K}=$ 0 uniformly in $u_{2}$ in each strip. The critical points of $\widetilde{K}$ are determined by the points $\left(u_{1}, u_{2}\right)$ which anihilate $f^{\prime}$ and $g^{\prime}$, which occur at $\widetilde{X}\left(0, u_{2}^{0}\right)$, where $u_{2}^{0}=m(2 k+1) \pi / 2 n, 0 \leq k \leq 2 n-1$. We conclude that the image of each of the $n$ regions of positive (resp. negative) curvature has an isolated point of maximum (resp. minimum) Gaussian curvature.

As an immediate consequence of $\widetilde{K}$, it follows that the total curvature vanishes. Moreover, a straightforward computation shows that on a horizontal strip with positive curvature, the total curvature is $4 \pi$. Hence, the total absolute curvature is $8 \pi n$.

In order to show that the ends $\mathcal{F}^{ \pm}$have geometric index $m$, we consider for each $\lambda \in R$, the intesection curve $\Gamma_{\lambda}$ of the surface with the horizontal plane $z=\lambda$. Let

$$
\gamma_{\lambda}=\left\{\left(u_{1}, u_{2}\right) \in R^{2} ; \widetilde{z}\left(u_{1}, u_{2}\right)=u_{1}+\frac{2 f^{\prime}(f+g)}{c\left[f^{2}-(1+2 \gamma) g^{2}\right]}=\lambda\right\} .
$$

It is not difficult to prove that $\gamma_{\lambda}$ is a regular connected curve, which is the graph of a function $u_{1}=\beta_{\lambda}\left(u_{2}\right)$, for $\lambda>0$ sufficiently large. Moreover, it follows from Proposition 2.4 that the curvature $k_{\lambda}$ of the curve $\Gamma_{\lambda}\left(u_{2}\right)=$ $\widetilde{X}\left(\beta_{\lambda}\left(u_{2}\right), u_{2}\right)$, has the following property: $\lim _{\lambda \rightarrow \infty} k_{\lambda}\left(u_{2}\right)=1$ uniformly in $u_{2}$. 
If $\sqrt{\xi(c, \gamma)}=n / m$ then $\widetilde{z}$ and hence $\beta_{\lambda}$ are periodic functions in $u_{2}$ with period $2 m \pi$. Therefore, for $\lambda$ sufficiently large, $\Gamma_{\lambda}$ is a closed curve and

$$
\lim _{\lambda \rightarrow \infty} \frac{1}{2 \pi} \int_{0}^{2 m \pi} k_{\lambda}\left(u_{2}\right) d u_{2}=m \text {. }
$$

The curve $\Gamma_{\lambda}$ will be a simple closed (convex) curve if and only if $m=1$. We conclude that the end $\mathcal{F}^{+}$has geometric index $m$ and it is embedded if and only if $m=1$. Using the symmetry of the surface $\left(\widetilde{X}_{c, \gamma}\left(-u_{1}, u_{2}\right)\right.$ is obtained by $\widetilde{X}_{c, \gamma}\left(u_{1}, u_{2}\right)$ by reflecting with respect to the $x 0 y$ plane) we get the result for $\mathcal{F}^{-}$.

If $c>0$ or $c<0$ and $\sqrt{\xi}$ is not a rational number then $\widetilde{X}_{c, \gamma}$ is a nonperiodic immersion of the plane into $R^{3}$, with an infinite number of isolated critical points of its Gaussian curvature.

Our next two results provide the symmetries of the complete linear Weingarten surfaces given by Proposition 2.3. In Figure 2, one can visualize some of the surfaces given by $\widetilde{X}_{c \gamma}$. In order to describe the symmetries of the surfaces we introduce the following notation:

A reflection with respect to a plane $z=z_{0}$ will be denoted by

$$
Z_{z_{0}}(x, y, z)=\left(x, y,-z+2 z_{0}\right) .
$$

Let $V_{\beta}=(-\sin \beta, \cos \beta, 0)$ be a unit vector determined by a constant $\beta$. The reflection with respect to the plane orthogonal to $V_{\beta}$ which passes through the origin is denoted by

$$
S_{\beta}(p)=p-2\left\langle p, V_{\beta}\right\rangle V_{\beta}, \quad p \in R^{3},
$$

where $\langle$,$\rangle denotes the euclidean inner product of R^{3}$.

Proposition 2.6. Any complete linear Weingarten surface $\widetilde{X}_{c \gamma}$, given by Proposition 2.3 with $c<0$ satisfies the following symmetries:

$$
\widetilde{X}_{c \gamma}\left(u_{1}, u_{2}+\theta\right)=R_{\theta} \widetilde{X}_{c \gamma}\left(u_{1}, u_{2}\right),
$$

where $\theta=2 \pi / \sqrt{\xi(c, \gamma)}$,

$$
\widetilde{X}_{c \gamma}\left(u_{1}, u_{2}+\beta_{k}\right)=S_{\beta_{k}} \widetilde{X}_{c \gamma}\left(u_{1},-u_{2}+\beta_{k}\right),
$$

where $\beta_{k}=(2 k+1) \pi / 2 \sqrt{\xi(c, \gamma)}$,

$$
\widetilde{X}_{c \gamma}\left(-u_{1}, u_{2}\right)=Z_{0} \tilde{X}_{c \gamma}\left(u_{1}, u_{2}\right),
$$

where $\xi$ is given by (44) and $R_{\theta}, Z_{0}$ and $S_{\beta_{k}}$ are defined by (50)-(62).

Proof. The surface $\widetilde{X}_{c \gamma}$ with $c<0$ is described by (43) where $f$ and $g$ are given by (53). Moreover, $\widetilde{X}_{c \gamma}\left(u_{1}, u_{2}\right)=R_{u_{2}} Y\left(u_{1}, u_{2}\right)$ where $Y=(1-$ $\left.g \Lambda,-g^{\prime} \Lambda, u_{1}-f^{\prime} \Lambda\right), \Lambda=(f+g) / c P$ and $P$ is given by (54). Therefore, we have

$$
\widetilde{X}_{c \gamma}\left(u_{1}, u_{2}+\theta\right)=R_{u_{2}+\theta} Y\left(u_{1}, u_{2}+\theta\right)=R_{\theta} R_{u_{2}} Y\left(u_{1}, u_{2}\right)=R_{\theta} \widetilde{X}_{c \gamma}\left(u_{1}, u_{2}\right),
$$




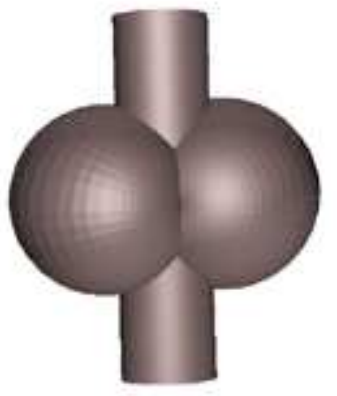

a)

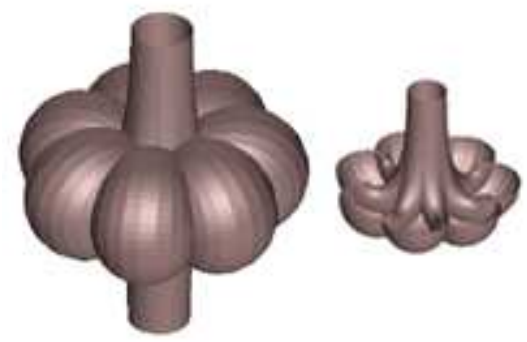

c)

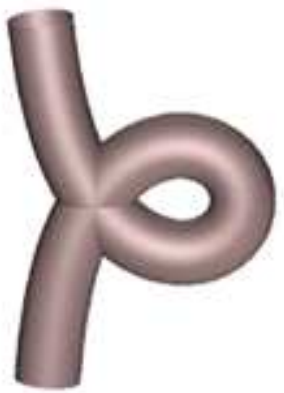

e)
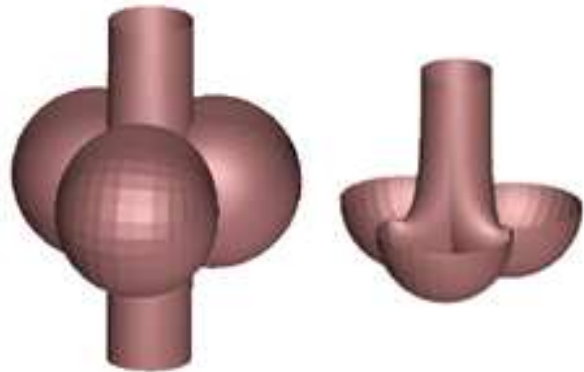

b)

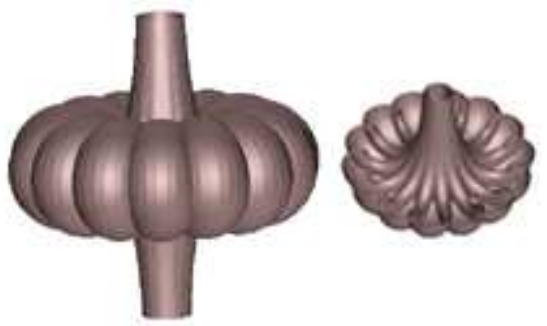

d)

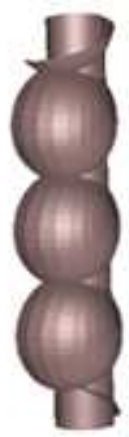

f)

Figure 2. Complete Weingarten surfaces $\widetilde{X}_{c \gamma}$ which satisfy the relation $-1 / 2+H+\gamma K=0$ and they are associated to the cylinder by Ribaucour transformations. a), b) and c) are 1-periodic cmc surfaces obtained by considering $\gamma=0$ and $\sqrt{1-c}=n / m$ a rational number equal to $2 / 1,3 / 2$ and $7 / 6$ respectively. d) and e) are 1-periodic Weingarten surfaces for which $\gamma=0.2, \sqrt{1-c(2 \gamma+1)}=14 / 13$ and $\gamma=-1 / 2, c=$ -0.1 respectively. f) is a cmc surface obtained by considering $c=2.8$ and $\gamma=0$. 
which proves (63).

From the definition of $S_{\beta_{k}}$ we have $S_{\beta_{k}}(x, y, z)=R_{2 \beta_{k}} S_{0}(x, y, z)$. Moreover, $g\left(-u_{2}+\beta_{k}\right)=g\left(u_{2}+\beta_{k}\right)$ and $g^{\prime}\left(-u_{2}+\beta_{k}\right)=-g^{\prime}\left(u_{2}+\beta_{k}\right)$. Hence,

$$
\begin{aligned}
S_{\beta_{k}} \widetilde{X}_{c \gamma}\left(u_{1},-u_{2}+\beta_{k}\right) & =R_{2 \beta_{k}} S_{0} R_{-u_{2}+\beta_{k}} Y\left(u_{1},-u_{2}+\beta_{k}\right) \\
& =R_{2 \beta_{k}} S_{0} R_{-u_{2}+\beta_{k}} S_{0} Y\left(u_{1}, u_{2}+\beta_{k}\right) \\
& =R_{u_{2}+\beta_{k}} Y\left(u_{1}, u_{2}+\beta_{k}\right)=\widetilde{X}_{c \gamma}\left(u_{1}, u_{2}+\beta_{k}\right),
\end{aligned}
$$

where we have used the identity $S_{0} R_{-u_{2}+\beta_{k}} S_{0}=R_{u_{2}-\beta_{k}}$. This proves (64). The proof of (65) follows from the equalities

$$
\widetilde{X}_{c \gamma}\left(-u_{1}, u_{2}\right)=R_{u_{2}} Y\left(-u_{1}, u_{2}\right)=Z_{0} \widetilde{X}_{c \gamma}\left(u_{1}, u_{2}\right) .
$$

Proposition 2.7. Any complete linear Weingarten surface $\widetilde{X}_{c \gamma}$, given by Proposition 2.3 with $c>0$ satisfies the following symmetries:

$$
\begin{aligned}
& \text { (66) } \widetilde{X}_{c \gamma}\left(u_{1}+\delta, u_{2}\right)=T_{\delta} \widetilde{X}_{c \gamma}\left(u_{1}, u_{2}\right), \quad \text { where } \delta=\frac{2 \pi}{\sqrt{c}}, \\
& \text { (67) } \widetilde{X}_{c \gamma}\left(u_{1}+z_{0}, u_{2}\right)=Z_{z_{0}} \widetilde{X}_{c \gamma}\left(-u_{1}+z_{0}, u_{2}\right), \quad \text { where } z_{0}=\frac{(2 k+1) \pi}{2 \sqrt{c}}, \\
& \text { (68) } \widetilde{X}_{c \gamma}\left(u_{1}, u_{2}\right)=S_{0} \widetilde{X}_{c \gamma}\left(u_{1},-u_{2}\right),
\end{aligned}
$$

where $k$ is an integer, $T_{\delta}, Z_{z_{0}}$ and $S_{0}$ are defined by (51), (61) and (62) respectively.

Proof. The surface $\widetilde{X}_{c \gamma}$ is described by (43) where $f$ and $g$ are given by (52). Therefore, (66) follows immediately. In order to prove (67), we observe that $f\left(-u_{1}+z_{0}\right)=f\left(u_{1}+z_{0}\right)$, and $f^{\prime}\left(u_{1}+z_{0}\right)=-f^{\prime}\left(-u_{1}+z_{0}\right)$. Therefore, we conclude that

$$
\tilde{X}_{c \gamma}\left(u_{1}+z_{0}, u_{2}\right)=R_{u_{2}} Y\left(u_{1}+z_{0}, u_{2}\right)=Z_{z_{0}} \tilde{X}_{c \gamma}\left(-u_{1}+z_{0}, u_{2}\right),
$$

where we have used the function $Y$ introduced in the proof of Proposition 2.6.

Finally, we prove (68) by observing that

$$
\begin{aligned}
S_{0} \widetilde{X}_{c \gamma}\left(u_{1},-u_{2}\right) & =S_{0} R_{-u_{2}} Y\left(u_{1},-u_{2}\right)=S_{0} R_{-u_{2}} S_{0} Y\left(u_{1}, u_{2}\right) \\
& =R_{u_{2}} Y\left(u_{1}, u_{2}\right)=R_{u_{2}} Y\left(u_{1}, u_{2}\right)=\widetilde{X}_{c \gamma}\left(u_{1}, u_{2}\right) .
\end{aligned}
$$

We conclude by observing that the linear Weingarten surfaces given by $\widetilde{X}_{c \gamma}$ are tubular surfaces when $\gamma=-1 / 2$, since they satisfy $\Delta=\beta^{2}-4 \alpha \gamma=$ 0 , and they provide examples of complete surfaces with $\Delta<0$ and $\Delta>0$. The lines of curvature $\widetilde{X}_{c \gamma}\left(u_{1}, u_{2}^{0}\right)$ are planar, while the curves $\widetilde{X}_{c \gamma}\left(u_{1}^{0}, u_{2}\right)$, when $f^{\prime}\left(u_{1}^{0}\right) \neq 0$, are contained on a sphere centered at $\left(0,0, f / f^{\prime}\left(u_{1}^{0}\right)\right)$ with radius $\sqrt{1+\left(f / f^{\prime}\right)^{2}}$. Whenever $f^{\prime}\left(u_{1}^{0}\right)=0$ (it can only occur for $c>0$ ) 
then $\widetilde{X}_{c \gamma}\left(u_{1}^{0}, u_{2}\right)$ are planar lines of curvature. Surfaces with one family of planar curvature lines while the other family of curvature lines is spherical is said to be of Joachimsthal type by Wente [W2].

\section{Families of cmc surfaces associated to the cylinder.}

In this section we describe a one parameter family of $1 / 2$-cmc surfaces obtained from the cylinder by Ribaucour transformations. The surfaces are contained in the class of linear Weingarten surfaces described in Proposition 2.1 where we restrict $\gamma=0$. These surfaces could also be obtained directly from the cylinder by applying Ribaucour transformations as in Corollaries 1.9 and 1.10 .

In [PS], Pinkal and Sterling introduced the notion of a solution of finite type of the equation $w_{z \bar{z}}+\sinh (2 w) / 2=0$, where $z$ is the complex variable $z=u_{1}+i u_{2}$. The cmc surfaces associated to such solutions, i.e., those parametrized by isothermal coordinates such that the metric is given by $d s^{2}=4 e^{2 w}\left(d u_{1}^{2}+d u_{2}^{2}\right)$, are also called cmc surfaces of finite type. In particular a solution $w$ is of finite type 1 , if considering $\varphi_{1}=w_{z}$ and $\varphi_{2}=w_{z z z}-2 w_{z}^{3}$, there exist complex numbers $a$ and $b$ such that $\varphi_{2}=a \varphi_{1}+b \bar{\varphi}_{1}$. We will show that the cmc surfaces we obtain associated to the cylinder are of finite type 1 .

Proposition 3.1. Excluding the cylinder, any $1 / 2$-cmc parametrized surface locally associated to the cylinder as in Theorem 1.5 is given, up to rigid motions of $R^{3}$, by

$$
\widetilde{X}_{c}=X+\frac{2}{c(f-g)}\left(f^{\prime} X_{u_{1}}+g^{\prime} X_{u_{2}}-g N\right)
$$

where $N$ is the inner unit normal field of the cylinder paramerized by (42), $c$ is a real constant such that $c<0$ or $c>1$, and the functions $f\left(u_{1}\right)$ and $g\left(u_{2}\right)$ are given by

$$
\begin{array}{cll}
f=\varepsilon_{1} \sqrt{c-1} \sin \left(\sqrt{c} u_{1}\right) \quad g=\varepsilon_{2} \sqrt{c} \cosh \left(\sqrt{c-1} u_{2}\right) & \text { if } c>1, \\
f=\varepsilon_{1} \sqrt{1-c} \cosh \left(\sqrt{|c|} u_{1}\right) g=\varepsilon_{2} \sqrt{|c|} \sin \left(\sqrt{1-c} u_{2}\right) & \text { if } c<0,
\end{array}
$$

where $\varepsilon_{i}= \pm 1$. These surfaces are of finite type 1 .

Proof. By considering $\gamma=0$ in (43)-(47), we get the right-hand side of (69), where $f$ and $g$ are solutions of

$$
f^{\prime \prime}+c f=0 \quad g^{\prime \prime}+(1-c) g=0
$$

and the initial conditions for $f$ and $g$ must satisfy

$$
\left(\left(f^{\prime}\right)^{2}+\left(g^{\prime}\right)^{2}+(1-c) g^{2}+c f^{2}\right)\left(u_{1}^{0}, u_{2}^{0}\right)=0 .
$$

Moreover, since this last condition should be identically satisfied by the nontrivial solution functions $f$ and $g$, we conclude that the constant $c \neq 0$ 
is such that $c(1-c) \leq 0$, hence $c<0$ or $c \geq 1$. However, when $c=1$, it follows from (73) that $g=b_{2}$ where $b_{2} \neq 0$ is a real constant and $f \equiv 0$. In this case $\widetilde{X}$ reduces to the cylinder and therefore $c \neq 1$.

From (44) we have that $\xi=1-c$. Therefore, using Corollary 2.2, we conclude that if $c>1$ then (52) reduces to (70) and if $c<0$ then (53) reduces to (71). Each 1/2-cmc surface described by (69) is parametrized by isothermal coordinates where the metric is given (see (49)) by $d s^{2}=$ $\psi^{2}\left(d u_{1}^{2}+d u_{2}^{2}\right)$, with $\psi=(f+g) /(g-f)$. Any $1 / 2$-cmc surface described above is of finite type 1 . In fact, considering $w=\log (\psi / 2), \varphi_{1}=w_{z}$, $\varphi_{2}=w_{z z z}-2 w_{z}^{3}$, it is a straightforward computation to verify that $\varphi_{2}=$ $(1 / 2-c) \varphi_{1}+\overline{\varphi_{1}}$.

The properties of these cmc surfaces are given in the following results:

Proposition 3.2. Any $1 / 2$-cmc surfaces $\widetilde{X}_{c}$ given by (69) is a complete surface asymptotically close to the cylinder. Moreover,

a) If $c<0$ and $\sqrt{1-c}=n / m$ is an irreducible rational number, then $\widetilde{X}_{c}$ is an immersion of a cylinder into $R^{3}$, with two ends of geometric index $m$ and $n$ isolated points of maximum (respectively minimum) for the Gaussian curvature. The total curvature of $\tilde{X}_{c}$ is zero, while its total absolute curvature is $8 \pi n$. The ends are embedded if and only if $m=1$. In this case they are cylindrical ends.

b) If $c>1$ or $c<0$ and $\sqrt{1-c}$ is not a rational number then $\tilde{X}_{c}$ is a nonperiodic immersion of $R^{2}$ into $R^{3}$, with an infinite number of isolated critical points of its Gaussian curvature.

Proof. The properties of any surface $\widetilde{X}_{c}$ and its asymptotic behaviour are consequences of Propositions 2.4 and 2.5, where we consider $\gamma=0$. We observe that in this case the Gaussian curvature $\widetilde{K}_{c}$ of $\widetilde{X}_{c}$, is given by $\widetilde{K}_{c}=$ $2 f g\left(f^{2}+g^{2}\right) /(f+g)^{4}$.

Proposition 3.3. Any $1 / 2$-cmc surface $\widetilde{X}_{c}$ with $c<0$ satisfies the symmetries defined by (63)-(65) where $\theta=2 \pi / \sqrt{1-c}$ and $\beta_{k}=(2 k+1) \pi /(2 \sqrt{1-c})$.

Proposition 3.4. Any $1 / 2$-cmc surface $\widetilde{X}_{c}$ with $c>1$ satisfies the symmetries defined by (66)-(68).

We observe that the family of cmc surfaces considered in this section, is a special case $(\gamma=0)$ of the linear Weingarten surfaces of the previous section. Therefore, they are cmc surfaces of Joachimsthal type (see $[\mathbf{A}, \mathbf{W a}]$ and $[\mathbf{W} 2])$.

\section{Families of cmc surfaces associated to Delaunay surfaces.}

In this section, by using Ribaucour transformations, we will obtain families of cmc surfaces associated to the Delaunay surfaces. We will consider the 
Delaunay surfaces parametrized by

$$
X\left(u_{1}, u_{2}\right)=\left(\rho\left(u_{1}\right) \cos \left(u_{2}\right), \rho\left(u_{1}\right) \sin \left(u_{2}\right), \varphi\left(u_{1}\right)\right),
$$

where $a \neq 0$ is a real constant such that $1-4 a H>0$,

$$
\begin{gathered}
\rho\left(u_{1}\right)=\frac{1}{\sqrt{2} H}\left(1-2 a H+\sqrt{1-4 a H} \cos \left(2 H u_{1}\right)\right)^{1 / 2}, \\
\varphi\left(u_{1}\right)=\int_{0}^{u_{1}}\left(H \rho+\frac{a}{\rho}\right) d t \quad u_{1} \in R .
\end{gathered}
$$

The generating curves are unduloids when $a>0$ and they are nodoids when $a<0$.

Proposition 4.1. A parametrized surface is a $H$-cmc surface locally associated to a Delaunay surface $X$, given by (74), by a Ribaucour transformation as in Corollary 1.10, if and only if, it is given by

$$
\begin{aligned}
\widetilde{X}=X-\frac{\rho}{c[a g+(\ell-H f) \rho]} & \\
& \cdot\left(\left(\rho^{\prime} g+f^{\prime}\right) X_{u_{1}}+\frac{g^{\prime}}{\rho} X_{u_{2}}-\left(\frac{a g}{\rho}+\ell+H \rho g\right) N\right)
\end{aligned}
$$

where $N$ is the inner unit normal vector field of the Delaunay surface, $c \neq \frac{1}{2 a}$ is such that $c<0$ or $c>2 H$ and $\ell\left(u_{1}\right), f\left(u_{1}\right), g\left(u_{2}\right)$ are solutions of the equations

$$
\begin{aligned}
f^{\prime \prime}-\left(c+\lambda^{1}\right) \ell-c \lambda^{2} f & =0, \\
g^{\prime \prime}+(1-2 a c) g & =0, \\
-\rho^{\prime} f^{\prime}+c \rho \lambda^{1} f+\rho\left(c+\lambda^{2}\right) \ell & =0,
\end{aligned}
$$

where

$$
\lambda^{1}=-H+a / \rho^{2} \quad \lambda^{2}=-H-a / \rho^{2}
$$

and the initial conditions must satisfy

$$
\left(\left(g^{\prime}\right)^{2}+(1-2 a c) g^{2}+\left(f^{\prime}\right)^{2}+\ell^{2}+2 c H f^{2}-2 c f \ell\right)\left(u_{1}^{0}, u_{2}^{0}\right)=0 .
$$

Proof. The first fundamental form of the Delaunay surface is given by $d s^{2}=$ $d u_{1}^{2}+\rho^{2}\left(u_{1}\right) d u_{2}^{2}$ and the eigenvalues $\lambda^{1}$ and $\lambda^{2}$ of $d N$ are given by (81). There are no umbilic points and the 1 -forms dual to the principal directions are $\omega_{1}=d u_{1} \omega_{2}=\rho d u_{2}$. Moreover, the connection forms are given by $\omega_{12}=\rho^{\prime} d u_{2}, \omega_{13}=-\lambda^{1} d u_{1}$ and $\omega_{23}=-\lambda^{2} \rho d u_{2}$. 
In order to obtain the Ribaucour transformations of the Delaunay surface, we need to solve the integrable system (16)-(18), and (37) (see Corollary 1.10), which reduces to the following system of differential equations:

(85) $\Omega_{1, u_{1}}=c \lambda^{2} \Omega+\left(c+\lambda^{1}\right) W$

$$
\begin{aligned}
\Omega_{, u_{1}}=\Omega_{1} & \Omega_{, u_{2}}=\Omega_{2} \rho \\
W_{, u_{1}}=-\Omega_{1} \lambda^{1} & W_{, u_{2}}=-\Omega_{2} \lambda^{2} \rho \\
\Omega+\left(c+\lambda^{1}\right) W & \Omega_{1, u_{2}}=\rho^{\prime} \Omega_{2} \\
\Omega_{2, u_{1}}=0 & \Omega_{2, u_{2}}=-\rho^{\prime} \Omega_{1}+c \rho \lambda^{1} \Omega+\rho\left(c+\lambda^{2}\right) W
\end{aligned}
$$

where the initial condition for the solution must satisfy (37) at a given point.

From (86) and (85) we get

$$
\Omega_{2}=g^{\prime}\left(u_{2}\right) \quad \Omega_{1}=\rho^{\prime} g+f^{\prime}\left(u_{1}\right)
$$

where $g$ and $f$ are functions of $u_{2}$ and $u_{1}$ respectively, and it follows from (83) and (84) that

$$
\Omega=\rho g+f \quad W=-\rho g \lambda^{2}+\ell\left(u_{1}\right),
$$

where

$$
\ell^{\prime}\left(u_{1}\right)=-\lambda^{1} f^{\prime}
$$

Substituting (87) and (88) into Equations (85) and (86), we get

$$
\begin{aligned}
& \left(\rho^{\prime \prime}+\rho \lambda^{1} \lambda^{2}\right) g=-f^{\prime \prime}+c \lambda^{2} f+\left(c+\lambda^{1}\right) \ell \\
& g^{\prime \prime}=\left(-\left(\rho^{\prime}\right)^{2}+c \rho^{2} \lambda^{1}-\rho^{2}\left(c+\lambda^{2}\right) \lambda^{2}\right) g-\rho^{\prime} f^{\prime}+c \rho \lambda^{1} f+\rho\left(c+\lambda^{2}\right) \ell .
\end{aligned}
$$

From the expression of $\rho$, we have $\rho^{\prime \prime}=-\frac{1}{\rho^{3}}\left(H^{2} \rho^{4}-a^{2}\right)$ and $-\left(\rho^{\prime}\right)^{2}+c \rho^{2} \lambda^{1}-$ $\rho^{2}\left(c+\lambda^{2}\right) \lambda^{2}=-1+2 a c \neq 0$.

On the other hand, using the expressions of $\lambda^{1}$ and $\lambda^{2}$, we have $\rho \lambda^{1} \lambda^{2}=$ $\left(H^{2} \rho^{4}-a^{2}\right) / \rho^{3}$. Therefore, we conclude that $f$ satisfies Equation (78) and

$$
\begin{aligned}
g^{\prime \prime}+(1-2 a c) g & =B \\
-\rho^{\prime} f^{\prime}+c \rho \lambda^{1} f+\rho\left(c+\lambda^{2}\right) \ell & =B
\end{aligned}
$$

where $B$ is a real constant, which we may consider, without loss of generality, to be zero. Hence $g$ satisfies $(79)$ and the functions $f\left(u_{1}\right), \ell\left(u_{1}\right)$ must satisfy (80). Moreover, from Corollary $1.10 c(c-2 H)>0$ and since $H>0$, it follows that $c<0$ or $c>2 H$.

Finally, from (87)-(88) and using the equalities $\left(\rho^{\prime}\right)^{2}+\rho^{2}\left(\lambda^{2}\right)^{2}=1$ and

$$
\rho^{\prime} f^{\prime}+c\left(\rho H-\frac{a}{\rho}\right) f-\rho\left(\lambda^{2}+c\right) \ell=0,
$$

we conclude that (37) reduces to (82).

We observe that in the open subset of $R^{2}$ where $c+\lambda^{2}$ does not vanish $\ell$ is defined by (80). Moreover, $\ell$ can be extended continuously to $R^{2}$. It is a straightforward computation to verify that $\ell$ satisfies (89). 
The cmc-surfaces given by (77) are called of Enneper type by Wente [W2], since one family of curvature lines is spherical. In fact, the curves $\widetilde{X}\left(u_{1}^{0}, u_{2}\right)$ are contained on a sphere centered at $\left(0,0, \varphi\left(u_{1}^{0}\right)-\phi\left(u_{1}^{0}\right)\right)$ with radius $\sqrt{\rho^{2}+\phi^{2}}$, where $\phi=\left[c \rho^{2}\left(\lambda^{1} f+\ell\right)-f\right] /\left(\rho^{\prime} \ell+\lambda^{2} \rho f^{\prime}\right)$.

The cmc surfaces described in Proposition 4.1 by $\tilde{X}$ depend on $\ell$ which is well-defined on $R^{2}$. However, the points where $c+\lambda^{2}=0$ may introduce singularities for the function $f$ which must satisfy (78). In the following result, we will restrict the range of the parameter $c$ providing a sufficient condition for the surfaces $\widetilde{X}$ to be defined on $R^{2}$.

Proposition 4.2. Let $\widetilde{X}\left(u_{1}, u_{2}\right)$ be an $H$-cmc surface locally associated to a Delaunay surface by a Ribaucour transformation as in Proposition 4.1. If c satisfies

$$
c<\frac{1}{2 a}-\frac{\sqrt{1-4 a H}}{2|a|}, \quad \text { or } \quad c>\frac{1}{2 a}+\frac{\sqrt{1-4 a H}}{2|a|}
$$

then $\widetilde{X}$ is defined on $R^{2}$ and it is given by $(77)$, where $f\left(u_{1}\right)$ is a solution of the equation

$$
\rho^{2}\left(c+\lambda^{2}\right) f^{\prime \prime}-\rho \rho^{\prime}\left(c+\lambda^{1}\right) f^{\prime}+2 a c(c-2 H) f=0
$$

$g\left(u_{2}\right)$ satisfies (79), $\ell\left(u_{1}\right)$ is given by (80), $\lambda^{1}$ and $\lambda^{2}$ are given by (81) and the initial conditions for $f$ and $g$ satisfy (82).

Proof. Since $c(c-2 H)>0$ we have $c \neq H$, and the hypothesis of $c$ satisfying $(90)$, is equivalent to

$$
\left|\frac{c-H-2 a c H}{(c-H) \sqrt{1-4 a H}}\right|>1 .
$$

This inequality occurs, if and only if, the function $c+\lambda^{2}$ does not vanish for any real value of $u_{1}$. Moreover, we observe that whenever $c$ satisfies the first inequality of (90) then $c+\lambda^{2}<0$ and if $c$ satisfies the second one then $c+\lambda^{2}>0$. It follows from (80) that the differential equation (78) for the function $f$ reduces to $(91)$.

In order to show that the H-cmc surfaces obtained in Proposition 4.2 are complete, we will need the following result, where we consider an assumption on the functions $f$ and $g$ which is equivalent to the non-vanishing of $S$ given by (21) (see Corollary 1.9).

Lemma 4.3. Let $f\left(u_{1}\right)$ and $g\left(u_{2}\right)$ be solutions of (91) and (79) respectively. Assume that $\rho g+f$ does not vanish, then: 
i) If $1-2 a c<0$ then

$$
\begin{aligned}
& f=c_{1} r\left(u_{1}\right) e^{i \alpha u_{1}}+c_{2} s\left(u_{1}\right) e^{-i \alpha u_{1}}, \\
& g=A \cosh \left(\sqrt{2 a c-1} u_{2}\right),
\end{aligned}
$$

where $A \neq 0$ and $\alpha$ are real numbers, $\alpha \neq k H, k \in \mathbf{Z} \backslash\{0\}, c_{1}, c_{2} \in \mathbf{C}$ and $r\left(u_{1}\right), s\left(u_{1}\right)$ are complex valued periodic functions of period $\pi / H$ or $2 \pi / H$.

ii) If $1-2 a c>0$ then

$$
\begin{aligned}
& f=c_{1} r\left(u_{1}\right) e^{\delta u_{1}}+c_{2} s\left(u_{1}\right) e^{-\delta u_{1}}, \\
& g=A \sin \left(\sqrt{1-2 a c} u_{2}\right),
\end{aligned}
$$

where $A \neq 0, c_{1}, c_{2}, \delta \neq 0$ are real numbers, $r\left(u_{1}\right), s\left(u_{1}\right)$ are real valued periodic functions of period $\pi / H$ such that for all $u_{1}, c_{1} r\left(u_{1}\right) c_{2} s\left(u_{1}\right)$ $>0$.

Proof. We start observing that $f$ is a solution of (91), which is of type $f^{\prime \prime}-\Lambda_{1} f^{\prime}-\Lambda_{2} f=0$, where $\Lambda_{1}$ and $\Lambda_{2}$ are real periodic functions of $u_{1}$, with period $\pi / H$ and $\Lambda_{1}$ is an odd function. Therefore, it follows from Floquet's theory $[\mathbf{L e}]$ that, given any real initial conditions, the only solution of this equation is real and it is of the form (92), (94) or of type

$$
f=c_{1} a\left(u_{1}\right)+c_{2}\left(u_{1} a\left(u_{1}\right)+b\left(u_{1}\right)\right),
$$

where $c_{1}, c_{2}$ are complex numbers and $r\left(u_{1}\right), s\left(u_{1}\right)$ are complex valued periodic functions of period $\pi / H$ or $2 \pi / H$. If $f$ is of type (96), then there exits $\left(u_{1}^{0}, u_{2}^{0}\right)$ which anihilates the function $\rho g+f$, which is a contradiction. It follows that $c_{2}=0$ and hence $f$ is of type (92) or (94).

Since $f$ and $g$ satisfy (82) in $R^{2}$, it follows from the expression of $\ell$ given by (80) that $f$ satisfies the equation

$$
\begin{aligned}
\left(1+h_{1}^{2}\right)\left(f^{\prime}\right)^{2}+2 h_{1} & \left(h_{2}-c\right) f f^{\prime} \\
& +\left[\left(h_{2}-c\right)^{2}-c(c-2 H)\right] f^{2}+A^{2}(1-2 a c)=0
\end{aligned}
$$

where

$$
h_{1}=\frac{\rho^{\prime}}{\rho\left(c+\lambda^{2}\right)} \quad \text { and } \quad h_{2}-c=\frac{-c(c-2 H)}{c+\lambda^{2}} .
$$

By considering (97) as a quadratic equation for $f^{\prime}$, its discriminant is given by

$$
\Delta=4(1-2 a c)\left[\frac{c(c-2 H) f^{2}}{\rho^{2}\left(c+\lambda^{2}\right)^{2}}-\left(1+h_{1}^{2}\right) A^{2}\right] \geq 0 .
$$

Now if $1-2 a c<0$, it follows from (98) that $f^{2} \leq A^{2}\left(\rho^{2}+\frac{1-2 a c}{c(c-2 H)}\right)$. Since the right-hand side of this inequality is a bounded function, we conclude that 
$f$ is bounded, hence it is of the form (92). Moreover, we can show that $g$ is given by (93).

If $1-2 a c>0$, then $f$ is of type (94). In fact, otherwise, $f$ is of the form (92) and it follows from Floquet's theory that there exists $u_{1}^{0}$ such that $f\left(u_{1}^{0}\right)=0$. Since $g(0)=0$, it follows that the pair $\left(u_{1}^{0}, 0\right)$ anihilates the function $\rho g+f$, which contradicts the hypothesis. Therefore, $f$ is of type (94) and for any integer $n$,

$$
f\left(u_{1}^{0}+n \frac{\pi}{H}\right)=c_{1} r\left(u_{1}^{0}\right) e^{\lambda u_{1}^{0}} e^{\lambda n \frac{\pi}{H}}+c_{2} s\left(u_{1}^{0}\right) e^{-\lambda u_{1}^{0}} e^{-\lambda n \frac{\pi}{H}} .
$$

We claim that $\forall u_{1} \in R$, we have $c_{1} r\left(u_{1}\right) c_{2} s\left(u_{1}\right)>0$. Otherwise, suppose there exits $u_{1}^{0}$ such that $r\left(u_{1}^{0}\right) s\left(u_{1}^{0}\right)=0$, then it follows from (99) that $f\left(u_{1}^{0}+\right.$ $n \pi / H)$ tends to zero when $n$ tends to $\pm \infty$. This is a contradiction because (98) implies that $f^{2} \geq A^{2}\left(\rho^{2}+\frac{1-2 a c}{c(c-2 H)}\right)$. Similarly, we get a contradiction if $c_{1} r\left(u_{1}^{0}\right) c_{2} s\left(u_{1}^{0}\right)<0$, since in this case, it follows from (94) that $f(R)=R$.

Proposition 4.4. Any surface of the family $\widetilde{X}$ given in Proposition 4.2 is a complete $H$-cmc surface. Moreover, if $1-2 a c>0$ and $\sqrt{1-2 a c}$ is an irreducible rational number, then $\widetilde{X}$ is an immersion of a cylinder into $R^{3}$, otherwise it is a immersion of $R^{2}$ into $R^{3}$.

Proof. The first fundamental form of any surface of the family $\widetilde{X}$ is given by (see Remark 1.11) $d s^{2}=\psi_{1}^{2} d u_{1}^{2}+\psi_{2}^{2} d u_{2}^{2}$ where

$$
\psi_{i}=\frac{a(\rho g+f) a_{i}}{a \rho g+\rho^{2}(\ell-H f)} \quad a_{1}=1, \text { and } a_{2}=\rho .
$$

Moreover, the functions $f$ and $\ell$ determined by (91) and (89) satisfy the following relation identically:

$$
(1-2 a c) A^{2}+\left(f^{\prime}\right)^{2}+2 c f(H f-\ell)+\ell^{2}=0 .
$$

i) If $1-2 a c>0$, then $f$ and $g$ are given by (94) (95). It follows from the above equation that $f$ and $H f-\ell$ do not vanish for any $u_{1} \in R$ and there exists $\varepsilon>0$ such that $|\ell / f-H| \geq \varepsilon$ for all $u_{1} \in R$. Using the arguments of the proof of Lemma 4.3, by considering Equation (97) as a quadratic equation for $f^{\prime} / f$, we get that $f^{\prime} / f$ is a bounded function and hence $\ell / f$ is also a bounded function. Hence,

$$
\lim _{\left|u_{1}\right| \rightarrow \infty}\left(\psi_{i}-\frac{a a_{i} f}{\rho^{2}(\ell-H f)}\right)=0
$$

uniformly in the variable $u_{2}$. Now, with arguments similar to those used in the proof of Proposition 2.5, we get $m>0$ such that $\left|\psi_{i}\right| \geq m$ for all $\left(u_{1}, u_{2}\right) \in R^{2}$ and hence we conclude that $\widetilde{X}$ is complete.

ii) If $1-2 a c<0$, then $f$ and $g$ are given by (92) and (93). Hence

$$
\lim _{\left|u_{2}\right| \rightarrow \infty}\left|\psi_{i}-a_{i}\right|=0
$$


unifomly in $u_{1}$. Therefore, there exists $m_{0}$ and $L$ positive real numbers such that $\left|\psi_{i}\right| \geq m_{0}$ for all $\left(u_{1}, u_{2}\right)$ such that $\left|u_{2}\right|>L$. One can show that for any $\alpha$, there exist $m_{i}>0$ such that $\left|\psi_{i}\left(u_{1}, u_{2}\right)\right| \geq m_{i}>0$ for all $\left(u_{1}, u_{2}\right) \in R \times[-L, L]$. We conclude that $\widetilde{X}$ is complete.

We conclude this section by observing that with the same arguments of the proof of Propositions 2.6 and 2.7, one can show that any H-cmc surface given in Proposition 4.2 satisfies the following symmetries:

$$
\begin{aligned}
& \tilde{X}\left(u_{1}, u_{2}+\theta\right)=R_{\theta} \tilde{X}\left(u_{1}, u_{2}\right) \quad \text { where } \theta=2 \pi / \sqrt{1-2 a c}, \\
& \widetilde{X}\left(u_{1}, u_{2}+\beta_{k}\right)=S_{\beta_{k}} \widetilde{X}\left(u_{1},-u_{2}+\beta_{k}\right) \quad \text { where } \beta_{k}=\frac{(2 k+1) \pi}{2 \sqrt{1-2 a c}}, k \in Z,
\end{aligned}
$$

whenever $1-2 a c>0$ and

$$
\widetilde{X}\left(u_{1}, u_{2}\right)=S_{0} \widetilde{X}\left(u_{1},-u_{2}\right)
$$

whenever $1-2 a c<0$.

One can also show that the complete H-cmc surfaces of the family $\widetilde{X}$ given by Proposition 4.1, whenever $1-2 a c<0$, are asymptotically close to the Delaunay surface. In fact, $\forall \varepsilon>0$ there exists $L>0$ such that

$$
\left|\tilde{X}\left(u_{1}, u_{2}\right)-X\left(u_{1}, u_{2} \pm \theta\right)\right|<\varepsilon \quad \forall\left(u_{1}, u_{2}\right) \in R^{2} \text { with } \pm u_{2} \geq L,
$$

where $\theta$ is such that $\cos \theta=1-1 /(a c)$ and $\sin \theta=-\sqrt{2 a c-1} /(a c)$.

\section{References}

[A] U. Abresch, Constant mean curvature tori in terms of elliptic functions, J. Reine Angew. Math., 374 (1987), 169-192, MR 88e:53006, Zbl 0597.53003.

[Bi] L. Bianchi, Lezioni di Geometria Differenciale, Bologna Nicola Zanichelli Ed., 1927.

[BS] F.B. Brito and R. Sa Earp, On the structure of certain Weingarten surfaces with boundary a circle, Ann. Fac. Sci. Toulouse, 6 (1997), 243-256, MR 99a:53005, Zbl 0901.53003.

[Bu] S.G. Buyske, Geometric aspects of Bäcklund transformations of Weingarten submanifolds, Pacific J. Math., 166 (1994), 213-223, MR 95m:53006, Zbl 0820.53004.

[CFT1] A.V. Corro, W. Ferreira and K. Tenenblat, On Ribaucour transformations for hypersurfaces, Mat. Contemp., 17 (1999), 137-160, MR 2002f:53104.

[CFT2] _ Minimal surfaces obtained by Ribaucour transformations, Geom. Dedicata, 96 (2003), 117-150, CMP 1956836.

[G-B] K. Große-Brauckmann, New surfaces of constant mean curvature, Math. Zeit., 214 (1993), 527-565, MR 94k:53012, Zbl 0806.53005.

[K] N. Kapouleas, Complete constant mean curvature surfaces in Euclidean three space, Ann. of Math., 131 (1990), 239-330, MR 93a:53007a, Zbl 0699.53007. 
[Ka] H. Karcher, The triply periodic minimal surfaces of A. Schoen and their constant mean curvature companions, Manuscripta Math., 64 (1989), 291-357, MR 90g:53010, Zbl 0687.53010.

[KKS] N. Korevaar, R. Kusner and B. Solomon, The structure of complete embedded surfaces with constant mean curvature, J. Differential Geom., 30 (1989), 465-503, MR 90g:53011, Zbl 0726.53007.

[L] B. Lawson, Complete minimal surfaces in $S^{3}$, Ann. of Math., 92 (1970), 335-374, MR 42 \#5170, Zbl 0205.52001.

[Le] S. Lefschetz, Differential Equations: Geometric Theory, Dover, 1977, MR 55 \#8441, Zbl 0107.07101.

[M] W.H. Meeks III, The topology and geometry of embedded surfaces of constant mean curvature, J. Differential Geom., 27 (1988), 539-552, MR 89h:53025, Zbl 0617.53007.

[PS] U. Pinkall and I. Sterling, On classification of constant mean curvature mean tori, Ann. of Math., 130 (1989), 407-451, MR 91b:53009, Zbl 0683.53053.

[RS] H. Rosenberg and R. Sa Earp, The geometry of properly embedded special surfaces in $R^{3}$; e.g., surfaces satisfying $a H+b K=1$, where $a$ and $b$ are positive, Duke Math. J., 73 (1994), 291-306, MR 95b:53010, Zbl 0802.53002.

[S] H. Sievert, Über die Zentralflächen der Enneperschen Flächen konstanten Krümmungsmasses, Diss. Tübingen, 1886.

[SW] I. Sterling and H.C. Wente, Existence and classification of cmc multibubbleton of finite and infinite type, Indiana Univ. Math. J., 42 (1993), 1239-1266, MR 95a:53015, Zbl 0803.53009.

[T] K. Tenenblat, Minimal and cmc surfaces obtained by Ribaucour transformations, Proceedings of the CMI Summer School, MSRI, to appear.

[Wa] R. Walter, Explicit examples to the H-problem of Heinz Hopf, Geom. Dedicata, 23 (1987), 187-213, MR 88i:53015a, Zbl 0699.53065.

[W1] H.C. Wente, Counterexample to a conjecture of H. Hopf, Pacific J. Math., 121 (1986), 193-243, MR 87d:53013, Zbl 0586.53003.

[W2] Constant mean curvature immersions of Enneper type, Memoirs Amer. Math. Soc., 100 (478) (1992), MR 93d:53015, Zbl 0772.53004.

Received June 12, 2002 and revised October 25, 2002. Partially supported by CNPq, PRONEX and PROCAD.

Instituto De Matemática e EstatísticA

UNIVERSidAde FEDERAL DE Goiás

74001-970 GOIÂNIA, GO

BRAZIL

E-mail address: corro@mat.ufg.br

Instituto De Matemática e Estatística

UNIVERSIDAde FEDERAL DE GoIÁS

74001-970 GOIÂNIA, GO

BRAZIL 
E-mail address: walter@mat.ufg.br

Departamento de Matemática

UNIVERSIDADE DE BRASÍLIA

70910-900, Brasília, DF

BRAZIL

E-mail address: keti@mat.unb.br 